\title{
An Intensity Standard for Electron Paramagnetic Resonance Using Chromium-Doped Corundum $\left(\mathrm{Al}_{2} \mathrm{O}_{3}: \mathrm{Cr}^{3+}\right)$
}

\author{
Te-Tse Chang, Donald Foster, ${ }^{*}$ and Arnold H. Kahn \\ Institute for Materials Research, National Bureau of Standards, Washington, D.C. 20234
}

(October 5, 1977)

\begin{abstract}
We report on the preparation of a standard reference material (SRM), made of single-crystal chromium-doped corundum (synthetic ruby), for use as an intensity standard in electron paramagnetic resonance (EPR) experiments. The SRM can be used to measure, by comparison, the number of spins in an unknown test sample.

We selected the above material for the standard because its magnetic resonance properties are well understood and because it is physically and chemically stable under common laboratory conditions. To prepare samples which gave satisfactory EPR signals, it was necessary to perform annealing and chemical etching after cutting. This removed strains and surface damage. After treatment, the $\mathrm{Cr}^{3+}$ resonance lines were sharper and the intensities were in good agreement with the theoretically predicted values. To aid in application, the theoretical resonance fields and line intensities were calculated and tabulated for arbitrary orientations of the sample, at several commonly used microwave frequencies. The concentration of $\mathrm{Cr}^{3+}$ in the samples was determined by measurement of the static susceptibility. A quantitative EPR intensity experiment, based on measuring the microwave power absorbed during resonance, gave the same concentration. This proves, that within experimental error the EPR experiment detects all the $\mathrm{Cr}^{3+}$ ions in the sample, which makes the ruby a useful SRM.

Key words: Absolute measurement; intensity standard; paramagnetic resonance; spin concentration; standard reference material (SRM); ruby.
\end{abstract}

\section{Introduction}

In applications of electron paramagnetic resonance in which a quantitative estimate of the number of spins is of importance there is need for standard laboratory samples. A preferred standard reference material for purposes of comparison of intensities should have a known number of identified spins, should be physically and chemically stable, and should have a narrow line spectrum which is usable over a wide temperature range. To meet this need we have produced samples of ruby, $\mathrm{Cr}^{3+}$ in aluminum oxide, suitable for laboratory use. This application has been suggested by Singer [1]. ${ }^{1}$ In this paper, we report on the research and testing performed for this application. Samples will be available under the Standard Reference Materials Program of NBS. Instructions for use will be supplied with the samples. These standard materials will have application in various fields, such as biological research in which spin concentrations change with time, in chemical kinetics, and in semiconductor doping. Under favorable conditions the user may expect to determine spin concentrations with a probable error in accuracy of \pm 10 percent.

Materials commonly used as standards for measurement of spin concentrations include free radicals, such as diphenyl-picryl-hydrazyl (DPPH) or $\gamma$-irradiated carbon; hydrated copper sulfate; and manganese chloride. All of these have utility but $\mathrm{Cr}^{3+}$ in aluminum oxide, ruby, may for many applications offer the best compromise of advantages over disadvantages.

In this paper we report on the research we performed for utilizing ruby as a standard. The spin-Hamiltonian parameters were remeasured and the resonance fields calculated for seven commonly used microwave frequencies. The relative intensities of the various absorption lines for the applied field parallel and perpendicular to the $c$ axis were found to be internally consistent within a probable error of \pm 5 percent. Absolute measurements of the number of $\mathrm{Cr}^{3+}$ spins in several ruby samples were obtained from the static

* Work performed while on sabbatical leave from Wichita State University, Wichita, KS 67208.

${ }^{1}$ Figures in brackets indicate the literature references at the end of this paper. 
magnetic susceptibility and by an absolute electron paramagnetic resonance method; the two agreed to within \pm 5 percent. This last agreement shows that within the accuracy of the experiment all chromium in the ruby specimens is in the $\mathrm{Cr}^{3+}$ valence state.

\section{Theoretical Background}

\subsection{Spin Hamiltonian}

The energy levels of the ground state of $\mathrm{Cr}^{3+}$ in $\mathrm{Al}_{2} \mathrm{O}_{3}$ placed in an external field $H$ are known to be describable by the spin Hamiltonian

$$
\mathscr{H}=g_{\|} \mu_{B} H_{z} S_{z}+g_{\perp} \mu_{B}\left(S_{x} H_{x}+S_{y} H_{y}\right)+D\left[S_{z}^{2}-(1 / 3) S(S+1)\right]
$$

In this form of the spin Hamiltonian the $z$ axis is taken along the $c$ axis of the crystal, and the $x$ and $y$ axes are normal to the $c$ axis, but arbitrarily oriented. The Bohr magneton is denoted by $\mu_{\mathrm{B}}$, the $g$ 's are the spectroscopic splitting factors and $D$ represents the crystalline field splitting. The spin angular momentum $S$ has the value $3 / 2$ (in units of $h$ ). The best numerical values of the constants at room temperature, as determined in this investigation are

$$
\begin{aligned}
g_{\|} & =1.9817 \pm 0.0004 \\
g_{\perp} & =1.9819 \pm 0.0006 \\
\frac{2 D}{h} & =-11.493 \pm 0.004 \mathrm{GHz}
\end{aligned}
$$

where the precision indices given represent probable errors. This spin Hamiltonian and the parameters were given by Manenkov and Prokhorov [2], by Geusic [3], Zaripov and Shamonin [4], and Kikuchi et al. [5]. The Hamiltonian is based upon the elementary expansion of the Hamiltonian in powers of the spin operators of the ground state of the $\mathrm{Cr}^{3+}$ ion subject to an axial crystalline environment. The most general Hamiltonian for spin $3 / 2$ in the $\mathrm{Al}_{2} \mathrm{O}_{3}$ environment $\left(\mathrm{C}_{3}\right.$ symmetry) was given by Statz and Koster [6] from group theoretical reasoning. Their description allows nine independent real constants in the Hamiltonian. However, in all work reported, including the present paper, the form eq (2.1), with three constants, gives an adequate description of the experimental EPR spectra of the ground state, and it is this form we shall use.

Numerical calculations of the spectrum of $\mathrm{Cr}^{3+}$ in $\mathrm{Al}_{2} \mathrm{O}_{3}$, as predicted by the Hamiltonian of eq (2.1) have been performed by W. S. Chang [7] and Schulz-du Bois [8]. These calculations verify the correctness of the model, but do not supply sufficient information for use of ruby as an intensity standard. For this reason we have recalculated the resonance fields and line intensities for several commonly used microwave frequencies.

To perform the necessary calculations we have rewritten the Hamiltonian of eq (2.1) in a laboratory system of reference axes, $x^{\prime}, y^{\prime}, z^{\prime}$, where the $z^{\prime}$ axis is along the static applied field $H_{0}$. Since the $\mathrm{Cr}^{3+}$ Hamiltonian possesses cylindrical symmetry about the $c$ axis of the host crystal, we are free to perform the calculations with the $y$ and $y^{\prime}$ axes coinciding. Then if the static field $H_{0}$ makes an angle $\theta$ with the $c$ axis, the laboratory coordinates $x^{\prime}, y^{\prime}, z^{\prime}$ of a point $x, y, z$ in the unprimed system are given by

$$
\begin{aligned}
& x^{\prime}=x \cos \theta+z \sin \theta \\
& y^{\prime}=y \\
& z^{\prime}=-x \sin \theta+z \cos \theta .
\end{aligned}
$$

The spin operators $S_{x}, S_{y}, S_{z}$ are transformed to $S_{x^{\prime}}, S_{y^{\prime}}, S_{z^{\prime}}$ in the same way as the coordinates. In this primed system the spin Hamiltonian becomes 


$$
\begin{aligned}
\mathscr{H}= & \mu_{B}\left(g_{\|} \cos ^{2} \theta+g_{\perp} \sin ^{2} \theta\right) H_{0} S_{z^{\prime}} \\
& +\mu_{B}\left(g_{\perp}-g_{\|}\right) \sin \theta \cos \theta H_{0^{\prime}} S_{x^{\prime}} \\
& +(1 / 2) D\left(3 \cos ^{2} \theta-1\right)\left[S_{z^{\prime}}^{2}-S(S+1) / 3\right] \\
& +D \sin \theta \cos \theta\left[S_{z^{\prime}} S_{x^{\prime}}+S_{x^{\prime}} S_{z^{\prime}}\right] \\
& +(1 / 2) D \sin ^{2} \theta\left[S_{x^{\prime}}^{2}-S_{y^{\prime}}^{2}\right] .
\end{aligned}
$$

This form of the Hamiltonian can be diagonalized readily in a basis system in which $S^{2}$ and $S_{z^{\prime}}$ are good quantum numbers. The difference of energy eigenvalues for a field $H_{0}$ and orientation $\theta$ represents a possible resonance absorption energy. The eigenvectors are determined as a step following the diagonalization and are needed in the calculation of the intensities. The details of the procedures for the calculations will be given in section 2.3. At this point it is sufficient to state that from the Hamiltonian of eq (2.6) we may obtain the solutions of the eigenvalue equation

$$
\mathscr{H} \psi_{\alpha}=E_{\alpha} \psi_{\alpha}
$$

where $E_{\alpha}$ are the energy eigenvalues and the vectors $\psi_{\alpha}$ are the associated eigenvectors which represent the spin states of the $\mathrm{Cr}^{3+}$ ion.

\subsection{Transition Probabilities and Line Intensities}

The paramagnetic resonance absorption is produced by application of a microwave radiation field $H_{1} \cos \omega t$, polarized normal to the static field $H_{0}$. This field induces transitions among the levels of the $\mathrm{Cr}^{3+}$ described in the previous section. The interaction $\mathscr{H}^{\prime}$ of the $\mathrm{Cr}^{3+}$ spin with the microwave field is of the magnetic dipole form and may be written

$$
\mathscr{H}^{\prime}=\left[g_{\perp} \mu_{B}\left(S_{x^{\prime}} H_{1 x^{\prime}}+S_{y^{\prime}} H_{1 y^{\prime}}\right)+g_{\|} \mu_{B} S_{z^{\prime}} H_{1 z^{\prime}}\right] \cos \omega t .
$$

We shall use eq (2.8) to calculate the rate of transitions and the absorption intensities. Since the quantities are seldom measured to better than a few percent in EPR experiments, we may in this part neglect the difference between $g_{\|}$and $g_{\perp}(\sim 0.01 \%)$ and work from the simpler form:

$$
\begin{aligned}
\mathscr{H}^{\prime} & =\bar{g} \mu_{B} H_{1} \cdot S \cos \omega t \\
& =\bar{g} \mu_{B} H_{1} S_{l} \cos \omega t
\end{aligned}
$$

where $\bar{g}$ is the average value given by

$$
\bar{g}=\left(g_{\|}+2 g_{\perp}\right) / 3
$$

and $S_{l}$ is the component $S$ along the direction of $H_{1}$. The usual experimental configuration has $H_{1}$ parallel to the $y^{\prime}$ axis. The transition rates induced by $\mathscr{H}^{\prime}$ may be calculated from the semiclassical radiation theory. Expanding on the treatment of Schiff [9], we obtain, for the time-proportional transitions between energy eigenstates $\psi_{\alpha}$ and $\psi_{\beta}$,

$$
w_{\alpha \beta}=\frac{\bar{g}^{2} \mu_{B}^{2}}{4 \hbar^{2}} H_{1}^{2}\left|\left\langle\alpha\left|S_{l}\right| \beta\right\rangle\right|^{2}\left[\delta\left(\frac{E_{\alpha}-E_{\beta}}{h}-\nu\right)+\delta\left(\frac{E_{\alpha}-E_{\beta}}{h}+\nu\right)\right] .
$$

This form of $w_{\alpha \beta}$ is based on the assumption that levels $\alpha$ and $\beta$ are well separated so that certain oscillatory perturbation terms connecting the levels may be neglected. The two delta functions of eq (2.11) correspond to 
the processes of absorption and stimulated emission. Proper use of eq (2.11) to obtain the total transition rate requires summing over final states and averaging over initial states. The levels will have slight local energy variations, but the other factors are essentially constant. The summing and averaging process is then equivalent to broadening each delta-function into a function $g_{\alpha \beta}(\nu)$, the line shape function for the $\alpha$ to $\beta$ transition, which has a peak in the vicinity of the average transition frequency and has unit integrated area. The transition rate per unit time, for absorption or stimulated emission, then becomes

$$
w_{\alpha \beta}=w_{\beta \alpha}=\frac{\bar{g}^{2} \mu_{B}^{2} H_{1}^{2}}{4 \hbar^{2}}\left|\left\langle\alpha\left|S_{l}\right| \beta\right\rangle\right|^{2} g_{\alpha \beta}(\nu) .
$$

The total energy absorption rate in the vicinity of the $\alpha$ to $\beta$ transition is

$$
p^{\alpha \beta}=w_{\alpha \beta}\left(N_{\alpha}-N_{\beta}\right) h \nu_{\alpha \beta}
$$

where $N_{\alpha}$ and $N_{\beta}$ are the populations of levels $\alpha$ and $\beta$. The total number of $\mathrm{Cr}^{3+}$ spins is $N_{0}$. For the high temperature limit, thermal equilibrium among the four levels of the $\mathrm{Cr}^{3+}$ spins give a population difference

$$
N_{\alpha}-N_{\beta}=\frac{N_{0}\left(E_{\alpha}-E_{\beta}\right)}{(2 S+1) k T}=\frac{N_{0} h \nu_{\alpha \beta}}{4 k T}
$$

which is valid for the temperature and frequencies of usual EPR conditions. The resultant absorption for all transitions may be written compactly as

$$
P_{a}(\nu)=\frac{N_{0} \pi^{2} \bar{g}^{2} \mu_{B}^{2} H_{1}^{2} \nu^{2}}{(2 S+1) k T} \sum_{\alpha<\beta}\left|\left\langle\alpha\left|S_{l}\right| \beta\right\rangle\right|^{2} g_{\alpha \beta}(\nu),
$$

where we have assumed that the individual absorption lines do not overlap. In the common EPR experiment the frequency is held fixed and the static field $H_{0}$ is swept slowly through the resonance, giving a field-sweep line shape $f\left(H_{0}\right)$. We may convert the field-sweep line shape to the frequency-sweep shape through the transformation

$$
g_{\alpha \beta}(\nu) d \nu=g_{\alpha \beta}(\nu) \frac{d \nu_{\alpha \beta}}{d H_{0}} d H_{0} .
$$

Thus, the field sweep shape function $F\left(H_{0}\right)$ may be identified as

$$
f_{\alpha \beta}\left(H_{0}\right)=g_{\alpha \beta}(\nu) \frac{d \nu_{\alpha \beta}}{d H_{0}} .
$$

For a narrow line $d \nu / d H_{0}$ is constant across the line profile. In this approximation, the absolute integrated intensity of a given transition may be obtained from eq (2.15) by using the field sweep line shape and dividing by the weighting factor $d v / d H_{0}$ for the particular transition.

\subsection{Method of Calculation}

The determination of the energy levels of the Hamiltonian of eq (2.6) can be performed by standard methods utilizing commercially available digital computer services. The Hamiltonian was written in a basis system spanned by the eigenstates of $S^{2}$ and $S_{z^{\prime}}$; i.e., the states $\mu_{m}$ such that

$$
S^{2} u_{m}=S(S+1) u_{m}=\frac{15}{4} \mu_{m}
$$

and

$$
S_{z^{\prime}} u_{m}=m u_{m}
$$


Here the states $u_{m}$ have $z^{\prime}$-component of angular momentum equal to $m$ (in units of $h$ ). The matrices for the operators in the expression for $\mathscr{H}$ are used in the explicit form given by Schiff, [10] in agreement with the Condon and Shortley [11] phase conventions.

The eigenstates of $\mathscr{H}, \psi_{\alpha}$, which represent the state of the $\mathrm{Cr}^{3+}$ spin, denoted by Greek subscripts, are the solutions of the equations

$$
\mathscr{H} \psi_{\alpha}=E_{\alpha} \psi_{\alpha}
$$

corresponding to energy eigenvalues $E_{\alpha}$. For each value of field $H_{0}$ and angle $\theta$ between $H_{0}$ and the $c$ axis there are four eigenvalues $E_{\alpha}$. Resonant transitions occur if a pair difference satisfies the resonance condition

$$
h \nu=E_{\alpha}-E_{\beta} .
$$

The eigenvectors $\psi_{\alpha}$ may be expanded i terms of the basic states $u_{m}$ (with Latin subscripts) in the form

$$
\psi_{\alpha}=\sum_{m} c_{\alpha o m} u_{m}
$$

The eigenvector components $c_{\alpha m}$ are obtained by standard subroutines when the eigenvalues are found. From the expressions for $\psi_{\alpha}$ it is then a simple matter to calculate the transition matrix element, $\left\langle\alpha\left|S_{l}\right| \beta\right\rangle$. In all cases we are interested in transitions induced by $H_{1}$ in the $x^{\prime}$ or $y^{\prime}$ directions only. The transition quantity needed for $y^{\prime}$-polarization, used in the most common experimental configuration,

$$
\left|\left\langle\alpha\left|S_{y},\right| \beta\right\rangle\right|^{2}
$$

may be obtained from application of eq $(2.22)$ to yield the expression

$$
\left\langle\alpha\left|S_{y^{\prime}}\right| \beta\right\rangle=\sum_{m, n} c_{\alpha m} c_{\beta n}\left\langle m\left|S_{y^{\prime}}\right| n\right\rangle
$$

where the elements $\left\langle m\left|S_{y^{\prime}}\right| n\right\rangle$ are well known in the basic system.

Finally, the weighting factor $d \nu_{\alpha \beta} / d H_{0}$ may be obtained in the following way. The Feynman-Hellmann theorem [12, 13] gives the derivative of the expectation value of the energy on a parameter of the Hamiltonian. Using this theorem we have, for any level $\alpha$,

$$
\frac{d E_{\alpha}}{d H_{0}}=\left\langle\alpha\left|\frac{\partial \mathscr{H}}{\partial H_{0}}\right| \alpha\right\rangle
$$

Now the required derivative is given by

$$
\frac{\partial \mathscr{H}}{\partial H_{0}}=\mu_{B}\left(g_{\|} \cos ^{2} \theta+g_{\perp} \sin ^{2} \theta\right) S_{z^{\prime}}
$$

The expectation value of this quantity can be evaluated easily when the eigenvectors $\psi_{\alpha}$ and $\psi_{\beta}$ are computed. The weighting factor is then given by

$$
\left|\frac{d \nu_{\alpha \beta}}{d H_{0}}\right|=\frac{\mu_{B}}{h}\left(g_{\|} \cos ^{2} \theta+g_{\perp} \sin ^{2} \theta\right)\left|\left\langle\alpha\left|S_{z^{\prime}}\right| \alpha\right\rangle-\left\langle\beta\left|S_{z^{\prime}}\right| \beta\right\rangle\right| .
$$

The explicit angular factor is almost constant for $\mathrm{Cr}^{3+}$ in ruby, but the difference of expectation values has a strong angular dependence and gives rise to a significant contribution to the line intensities.

This completes the description of the mathematical methods used to obtain all the necessary results. In the following section we shall describe the computational sequences used and the format of the tabulated results. 


\subsection{Tabulation of Results}

In the discussion that follows, the spin states and transitions will be labeled in the following systematic way: For an arbitrary orientation of the $c$ axis with respect to the field, the energy levels are identified as $\alpha$ $=1,2,3,4$, in sequence from the highest level downward. A particular resonance transition between levels $\alpha$ and $\beta$ is identified by the symbol $(\alpha, \beta)$. For the special cases when the $c$ axis is parallel to the applied field $H_{0}$, the states may be labeled by the component of $S_{z}$, which can take on the values $3 / 2,1 / 2,-1 / 2,-3 / 2$ (which is not necessarily in the order of the energies). For this orientation only, the transitions will sometimes be labeled $(3 / 2,1 / 2)$, etc. No ambiguity should result, as the half-integer notation is used only when the $c$ axis is parallel to $H_{0}$.

To obtain a preliminary estimate of the location of the resonances, the spin Hamiltonian of eq (2.6) was diagonalized for fields $H_{0}$ from 0 Oe to $5000 \mathrm{Oe}$ in steps of $100 \mathrm{Oe}$, and from $5000 \mathrm{Oe}$ to 25,000 Oe in steps of $500 \mathrm{Oe}$. This was performed for angles $\theta=0^{\circ}$ to $90^{\circ}$, at every $10^{\circ}$. This process enabled us to locate coarsely all observable transitions at frequencies as high as $50 \mathrm{GHz}$. We then applied a computational search procedure which located precisely the resonance fields for seven commonly used microwave frequencies, 6.0, $9.5,14.5,18.0,24.0,35.0$, and $50.0 \mathrm{GHz}$. The resonance fields $H_{0}$ are presented, respectively, in table 1 through table 7 , each table giving the results for a particular frequency at the ten orientations of the crystalline $c_{0}$ axis with respect to the static field $H_{0}$. Each resonance is identified by the two levels involved in the transition by the symbols $(\alpha, \beta)$. The numbering convention used as described in the preceding paragraph, is to label the levels as $1,2,3,4$ in sequence, from the highest energy level downwards. The results within a table are grouped together for a given angle $\theta$.

The transition probabilities and integrated frequency-sweep intensities for $x^{\prime}$ and $y^{\prime}$ polarizations are proportional to the quantities $T_{x^{\prime}}$ and $T_{y^{\prime}}$, which are given by

$$
\begin{aligned}
& T_{x^{\prime}}^{\alpha \beta}=\left|\left\langle\alpha\left|S_{x^{\prime}}\right| \beta\right\rangle\right|^{2} \\
& T_{y^{\prime}}^{\alpha \beta}=\left|\left\langle\alpha\left|S_{y^{\prime}}\right| \beta\right\rangle\right|^{2} .
\end{aligned}
$$

These quantities are tabulated in the columns of table 1 through table 7 following the transition identification. The field-sweep intensities are proportional to the T's divided by the weighting factors for the transitions. We define dimensionless field sweep quantities $U_{x^{\prime}}^{\alpha \beta}$ and $U_{y^{\prime}}^{\alpha \beta}$ by the relation,

$$
U_{x^{\prime}}^{\alpha \beta}=\frac{T_{x^{\prime}}^{\alpha \beta}}{\left|\frac{d \nu_{\alpha \beta}}{d H_{0}}\right|}\left(\frac{g_{\|} \mu_{B}}{h}\right)
$$

and similarly for $U_{y^{\prime}}^{\alpha \beta}$. The quantities $U_{x^{\prime}}^{\alpha \beta}$ and $U_{y^{\prime}}^{\alpha \beta}$ are listed in the last two columns of tables 1-7. For $c$ parallel $H_{0}$ each $T$ and the corresponding $U$ are numerically equal. Thus, the effect of the weighting factor at any angle can be seen by comparison of $T$ and $U$.

The values of the resonance field $H_{0}$ as a function of the angle $\theta$ between the $c$ axis and $H_{0}$ are plotted in figures 1 through 7 . Each figures gives the resonance fields for a particular microwave frequency.

\subsection{Intensity of Absorption}

The power absorption of an EPR resonance line is obtained through the measurement of the quality factor, $Q$, of the cavity system with the sample. In principle, an absolute measurement could be performed with any 
sample. Then if certain requirements are met, principally that of a narrow line spectrum, it would be possible to obtain an absolute measurement of the number of spins. A simpler method is to compare the resonances of the unknown sample with that of a standard sample having a known spin concentration, simultaneously placed in the cavity. From the ratio of the integrated intensities of the two resonances it is then possible to obtain the spin concentration in the unknown sample. In this section the question of absolute and relative absorption intensities will be taken up and the method of comparison with a standard will be discussed.

TABLE 1. The resonance magnetic field, the transition probability, $T$, and the integrated intensity, $U$, of $\mathrm{Cr}^{3+}$ ion in ruby, for

\begin{tabular}{|c|c|c|c|c|c|c|}
\hline ANGLE (Deg) & $H(\mathrm{Oe})$ & TRANS. & $T_{x^{\prime}}$ & $T_{y^{\prime}}$ & $U_{x^{\prime}}$ & $U_{y^{\prime}}$ \\
\hline 0.0 & $\begin{array}{r}721.1 \\
990.2 \\
1980.5 \\
2163.3 \\
3153.5 \\
6306.6\end{array}$ & $\begin{array}{l}(3,4) \\
(2,3) \\
(1,3) \\
(1,3) \\
(2,3) \\
(1,2)\end{array}$ & $\begin{array}{r}0.000 \\
.000 \\
.750 \\
1.000 \\
0.000 \\
.750\end{array}$ & $\begin{array}{r}0.000 \\
.000 \\
.750 \\
1.000 \\
0.000 \\
.750\end{array}$ & $\begin{array}{r}0.000 \\
.000 \\
.750 \\
1.000 \\
0.000 \\
.750\end{array}$ & $\begin{array}{r}0.000 \\
.000 \\
.750 \\
1.000 \\
0.000 \\
.750\end{array}$ \\
\hline 10.0 & $\begin{array}{r}732.6 \\
996.5 \\
2076.6 \\
2143.7 \\
3263.7 \\
5737.3\end{array}$ & $\begin{array}{l}(3,4) \\
(2,3) \\
(1,2) \\
(1,2) \\
(2,3) \\
(1,2)\end{array}$ & $\begin{array}{l}.000 \\
.035 \\
.162 \\
.051 \\
.261 \\
.501\end{array}$ & $\begin{array}{r}.000 \\
.037 \\
1.630 \\
1.441 \\
.249 \\
.763\end{array}$ & $\begin{array}{l}.000 \\
.018 \\
.311 \\
.113 \\
.149 \\
.608\end{array}$ & $\begin{array}{r}.000 \\
.019 \\
3.124 \\
3.180 \\
0.142 \\
.926\end{array}$ \\
\hline 20.0 & $\begin{array}{r}768.9 \\
1019.5 \\
1850.0 \\
3454.3\end{array}$ & $\begin{array}{l}(3,4) \\
(2,3) \\
(1,2) \\
(2,3)\end{array}$ & $\begin{array}{l}.000 \\
.096 \\
.458 \\
.539\end{array}$ & $\begin{array}{r}.000 \\
.123 \\
1.235 \\
0.478\end{array}$ & $\begin{array}{l}.000 \\
.051 \\
.417 \\
.362\end{array}$ & $\begin{array}{r}.000 \\
.065 \\
1.124 \\
0.320\end{array}$ \\
\hline 30.0 & $\begin{array}{r}837.1 \\
1072.7 \\
1643.0 \\
3545.3\end{array}$ & $\begin{array}{l}(3,4) \\
(2,3) \\
(1,2) \\
(2,3)\end{array}$ & $\begin{array}{l}.001 \\
.120 \\
.407 \\
.659\end{array}$ & $\begin{array}{r}.001 \\
.224 \\
1.159 \\
0.582\end{array}$ & $\begin{array}{l}.000 \\
.071 \\
.326 \\
.505\end{array}$ & $\begin{array}{l}.000 \\
.132 \\
.930 \\
.446\end{array}$ \\
\hline 40.0 & $\begin{array}{r}952.3 \\
1178.1 \\
1473.0 \\
3416.0\end{array}$ & $\begin{array}{l}(3,4) \\
(2,3) \\
(1,2) \\
(2,3)\end{array}$ & $\begin{array}{l}.003 \\
.085 \\
.364 \\
.615\end{array}$ & $\begin{array}{r}.003 \\
.325 \\
1.124 \\
0.628\end{array}$ & $\begin{array}{l}.001 \\
.061 \\
.265 \\
.565\end{array}$ & $\begin{array}{l}.001 \\
.233 \\
.817 \\
.577\end{array}$ \\
\hline 50.0 & $\begin{array}{l}1150.4 \\
1343.7 \\
1407.0 \\
2947.7\end{array}$ & $\begin{array}{l}(3,4) \\
(1,2) \\
(2,3) \\
(2,3)\end{array}$ & $\begin{array}{l}.011 \\
.335 \\
.015 \\
.392\end{array}$ & $\begin{array}{r}.012 \\
1.104 \\
0.434 \\
.638\end{array}$ & $\begin{array}{l}.006 \\
.225 \\
.017 \\
.530\end{array}$ & $\begin{array}{l}.007 \\
.742 \\
.490 \\
.862\end{array}$ \\
\hline 60.0 & $\begin{array}{l}1250.4 \\
1526.6\end{array}$ & $\begin{array}{l}(1,2) \\
(3,4)\end{array}$ & $\begin{array}{l}.317 \\
.050\end{array}$ & $\begin{array}{l}1.092 \\
0.047\end{array}$ & $\begin{array}{l}.200 \\
.040\end{array}$ & $\begin{array}{l}.690 \\
.038\end{array}$ \\
\hline 70.0 & $\begin{array}{l}1187.9 \\
2366.4\end{array}$ & $\begin{array}{l}(1,2) \\
(3,4)\end{array}$ & $\begin{array}{l}.307 \\
.290\end{array}$ & $\begin{array}{l}1.085 \\
0.174\end{array}$ & $\begin{array}{l}.186 \\
.372\end{array}$ & $\begin{array}{l}.656 \\
.223\end{array}$ \\
\hline 80.0 & $\begin{array}{l}1151.6 \\
3565.6\end{array}$ & $\begin{array}{l}(1,2) \\
(3,4)\end{array}$ & $\begin{array}{l}.302 \\
.935\end{array}$ & $\begin{array}{l}1.082 \\
0.321\end{array}$ & $\begin{array}{r}.178 \\
1.138\end{array}$ & $\begin{array}{l}.637 \\
.391\end{array}$ \\
\hline 90.0 & $\begin{array}{l}1139.8 \\
4048.4\end{array}$ & $\begin{array}{l}(1,2) \\
(3,4)\end{array}$ & $\begin{array}{r}.300 \\
1.262\end{array}$ & $\begin{array}{l}1.081 \\
0.360\end{array}$ & $\begin{array}{l}0.175 \\
1.377\end{array}$ & $\begin{array}{l}.630 \\
.393\end{array}$ \\
\hline
\end{tabular}


TABLE 2. The resonance magnetic field, the transition probability, $T$, and the integrated intensity, $U$, of $\mathrm{Cr}^{3+}$ ion in ruby, for

\begin{tabular}{|c|c|c|c|c|c|c|}
\hline ANGLE (Deg) & $H(\mathrm{Oe})$ & TRANS. & $T_{x^{\prime}}$ & $T_{y^{\prime}}$ & $U_{x^{\prime}}$ & $U_{y^{\prime}}$ \\
\hline 0.0 & $\begin{array}{r}359.4 \\
718.7 \\
1141.8 \\
3425.0 \\
3784.4 \\
7568.8\end{array}$ & $\begin{array}{l}(2,3) \\
(1,3) \\
(3,4) \\
(1,3) \\
(2,3) \\
(1,2)\end{array}$ & $\begin{array}{r}0.000 \\
.750 \\
.000 \\
1.000 \\
0.000 \\
.750\end{array}$ & $\begin{array}{r}0.000 \\
.750 \\
.000 \\
1.000 \\
0.000 \\
.750\end{array}$ & $\begin{array}{r}0.000 \\
.750 \\
.000 \\
1.000 \\
0.000 \\
.750\end{array}$ & $\begin{array}{r}0.000 \\
.750 \\
.000 \\
1.000 \\
0.000 \\
.750\end{array}$ \\
\hline 10.0 & $\begin{array}{r}360.2 \\
760.2 \\
1160.9 \\
3146.1 \\
4042.6 \\
7154.3\end{array}$ & $\begin{array}{l}(2,3) \\
(1,3) \\
(3,4) \\
(1,3) \\
(2,3) \\
(1,2)\end{array}$ & $\begin{array}{l}.025 \\
.688 \\
.000 \\
.793 \\
.541 \\
.646\end{array}$ & $\begin{array}{l}.026 \\
716 \\
.000 \\
.738 \\
.526 \\
.759\end{array}$ & $\begin{array}{l}.012 \\
.737 \\
.000 \\
.645 \\
.368 \\
.692\end{array}$ & $\begin{array}{l}.013 \\
.768 \\
.000 \\
.600 \\
.358 \\
.813\end{array}$ \\
\hline 20.0 & $\begin{array}{r}363.7 \\
920.3 \\
1221.9 \\
2730.5 \\
4351.6 \\
5948.2\end{array}$ & $\begin{array}{l}(2,3) \\
(1,3) \\
(3,4) \\
(1,3) \\
(2,3) \\
(1,2)\end{array}$ & $\begin{array}{l}.074 \\
.526 \\
.001 \\
.701 \\
.705 \\
.393\end{array}$ & $\begin{array}{l}.087 \\
.622 \\
.001 \\
.397 \\
.656 \\
.796\end{array}$ & $\begin{array}{l}.038 \\
.729 \\
.000 \\
.528 \\
.532 \\
.531\end{array}$ & $\begin{array}{l}.044 \\
.861 \\
.000 \\
.299 \\
.495 \\
1.076\end{array}$ \\
\hline 30.0 & $\begin{array}{r}373.0 \\
1338.7 \\
4081.2 \\
4532.8\end{array}$ & $\begin{array}{l}(2,3) \\
(3,4) \\
(1,2) \\
(2,3)\end{array}$ & $\begin{array}{l}.111 \\
.007 \\
.151 \\
.772\end{array}$ & $\begin{array}{l}.157 \\
.007 \\
.927 \\
.706\end{array}$ & $\begin{array}{l}.059 \\
.003 \\
.330 \\
.622\end{array}$ & $\begin{array}{r}0.083 \\
.003 \\
2.032 \\
.568\end{array}$ \\
\hline 40.0 & $\begin{array}{r}391.4 \\
1544.9 \\
2689.1 \\
4519.5\end{array}$ & $\begin{array}{l}(2,3) \\
(3,4) \\
(1,2) \\
(2,3)\end{array}$ & $\begin{array}{l}.116 \\
.028 \\
.201 \\
.773\end{array}$ & $\begin{array}{r}.225 \\
.031 \\
1.233 \\
0.735\end{array}$ & $\begin{array}{l}.065 \\
.014 \\
.299 \\
.667\end{array}$ & $\begin{array}{r}.127 \\
.015 \\
1.828 \\
0.634\end{array}$ \\
\hline 50.0 & $\begin{array}{r}422.7 \\
1929.3 \\
2331.2 \\
4277.0\end{array}$ & $\begin{array}{l}(2,3) \\
(3,4) \\
(1,2) \\
(2,3)\end{array}$ & $\begin{array}{l}.093 \\
.097 \\
.300 \\
.709\end{array}$ & $\begin{array}{r}.287 \\
.103 \\
1.213 \\
0.756\end{array}$ & $\begin{array}{l}.058 \\
.068 \\
.287 \\
.673\end{array}$ & $\begin{array}{r}.180 \\
.072 \\
1.160 \\
0.717\end{array}$ \\
\hline 60.0 & $\begin{array}{r}475.0 \\
2147.7 \\
2716.4 \\
3805.5\end{array}$ & $\begin{array}{l}(2,3) \\
(1,2) \\
(3,4) \\
(2,3)\end{array}$ & $\begin{array}{l}.053 \\
.337 \\
.293 \\
.595\end{array}$ & $\begin{array}{r}.346 \\
1.169 \\
0.251 \\
.779\end{array}$ & $\begin{array}{l}.039 \\
.275 \\
.321 \\
.647\end{array}$ & $\begin{array}{l}.254 \\
.953 \\
.276 \\
.847\end{array}$ \\
\hline 70.0 & $\begin{array}{r}564.8 \\
2035.9 \\
3143.7 \\
3965.6\end{array}$ & $\begin{array}{l}(2,3) \\
(1,2) \\
(2,3) \\
(3,4)\end{array}$ & $\begin{array}{l}.014 \\
.355 \\
.456 \\
.649\end{array}$ & $\begin{array}{r}.409 \\
1.142 \\
0.812 \\
.382\end{array}$ & $\begin{array}{l}.014 \\
.267 \\
.611 \\
.790\end{array}$ & $\begin{array}{r}.386 \\
.860 \\
1.087 \\
0.465\end{array}$ \\
\hline 80.0 & $\begin{array}{r}750.0 \\
1973.4 \\
2335.9 \\
5006.2\end{array}$ & $\begin{array}{l}(2,3) \\
(1,2) \\
(2,3) \\
(3,4)\end{array}$ & $\begin{array}{l}.002 \\
.363 \\
.318 \\
.984\end{array}$ & $\begin{array}{r}.504 \\
1.129 \\
0.878 \\
.437\end{array}$ & $\begin{array}{r}.002 \\
.262 \\
.640 \\
1.075\end{array}$ & $\begin{array}{r}.784 \\
.815 \\
1.769 \\
0.478\end{array}$ \\
\hline 90.0 & $\begin{array}{l}1953.5 \\
5386.7\end{array}$ & $\begin{array}{l}(1,2) \\
(3,4)\end{array}$ & $\begin{array}{r}.366 \\
1.117\end{array}$ & $\begin{array}{l}1.124 \\
0.452\end{array}$ & $\begin{array}{l}0.261 \\
.1 .162\end{array}$ & $\begin{array}{l}.802 \\
.470\end{array}$ \\
\hline
\end{tabular}


TABLE 3. The resonance magnetic field, the transition probability, $\mathrm{T}$, and the integrated intensity, $\mathrm{U}$, of $\mathrm{Cr}^{3+}$ ion in ruby, for microwave frequency of $14.500 \mathrm{GHz}$

\begin{tabular}{|c|c|c|c|c|c|c|}
\hline ANGLE (Deg) & $H(\mathrm{Oe})$ & TRANS. & $T_{x^{\prime}}$ & $T_{y^{\prime}}$ & $U_{x^{\prime}}$ & $U_{y^{\prime}}$ \\
\hline 0.0 & $\begin{array}{r}542.2 \\
1084.4 \\
1742.6 \\
4685.9 \\
5227.5 \\
9371.6\end{array}$ & $\begin{array}{l}(1,4) \\
(2,4) \\
(3,4) \\
(1,3) \\
(2,3) \\
(1,2)\end{array}$ & $\begin{array}{r}0.000 \\
.750 \\
.000 \\
.000 \\
1.000 \\
0.750\end{array}$ & $\begin{array}{r}0.000 \\
.750 \\
.000 \\
.000 \\
1.000 \\
0.750\end{array}$ & $\begin{array}{r}0.000 \\
.750 \\
.000 \\
.000 \\
1.000 \\
0.750\end{array}$ & $\begin{array}{r}0.000 \\
.750 \\
.000 \\
.000 \\
1.000 \\
0.750\end{array}$ \\
\hline 10.0 & $\begin{array}{r}540.6 \\
1123.4 \\
1776.0 \\
4398.4 \\
5482.9 \\
9038.1\end{array}$ & $\begin{array}{l}(1,4) \\
(2,4) \\
(3,4) \\
(1,3) \\
(2,3) \\
(1,2)\end{array}$ & $\begin{array}{l}.016 \\
.724 \\
.003 \\
.332 \\
.893 \\
.703\end{array}$ & $\begin{array}{l}.017 \\
.736 \\
.003 \\
.322 \\
.880 \\
.757\end{array}$ & $\begin{array}{l}.008 \\
.774 \\
.001 \\
.198 \\
.801 \\
.723\end{array}$ & $\begin{array}{l}.008 \\
.756 \\
.001 \\
.193 \\
.790 \\
.778\end{array}$ \\
\hline 20.0 & $\begin{array}{r}538.7 \\
1240.6 \\
1891.0 \\
3941.4 \\
5822.8 \\
8107.4\end{array}$ & $\begin{array}{l}(1,4) \\
(2,4) \\
(3,4) \\
(1,3) \\
(2,3) \\
(1,2)\end{array}$ & $\begin{array}{l}.050 \\
.663 \\
.044 \\
.391 \\
.867 \\
.583\end{array}$ & $\begin{array}{l}.055 \\
.707 \\
.045 \\
.333 \\
.833 \\
.779\end{array}$ & $\begin{array}{l}.024 \\
.721 \\
.017 \\
.240 \\
.753 \\
.650\end{array}$ & $\begin{array}{l}.027 \\
.769 \\
.018 \\
.205 \\
.724 \\
.869\end{array}$ \\
\hline 30.0 & $\begin{array}{r}539.8 \\
1425.8 \\
2149.6 \\
3455.9 \\
6048.8 \\
6794.9\end{array}$ & $\begin{array}{l}(1,4) \\
(2,4) \\
(3,4) \\
(1,3) \\
(2,3) \\
(1,2)\end{array}$ & $\begin{array}{l}.077 \\
.590 \\
.182 \\
.342 \\
.870 \\
.452\end{array}$ & $\begin{array}{l}.097 \\
.672 \\
.195 \\
.198 \\
.825 \\
.827\end{array}$ & $\begin{array}{l}.038 \\
.650 \\
.102 \\
.210 \\
.760 \\
.560\end{array}$ & $\begin{array}{r}.047 \\
.741 \\
.109 \\
.121 \\
.721 \\
1.026\end{array}$ \\
\hline 40.0 & $\begin{array}{r}548.8 \\
1646.1 \\
2681.2 \\
2914.5 \\
5457.0 \\
6099.6\end{array}$ & $\begin{array}{l}(1,4) \\
(2,4) \\
(3,4) \\
(1,3) \\
(1,2) \\
(2,3)\end{array}$ & $\begin{array}{l}.088 \\
.505 \\
.340 \\
.218 \\
.372 \\
.866\end{array}$ & $\begin{array}{l}.134 \\
.621 \\
.359 \\
.028 \\
.911 \\
.831\end{array}$ & $\begin{array}{l}.043 \\
.506 \\
.297 \\
.145 \\
.494 \\
.773\end{array}$ & $\begin{array}{r}.065 \\
.623 \\
.313 \\
.018 \\
1.208 \\
.742\end{array}$ \\
\hline 50.0 & $\begin{array}{r}568.4 \\
1883.2 \\
2295.3 \\
3599.2 \\
4466.4 \\
5953.1\end{array}$ & $\begin{array}{l}(1,4) \\
(2,4) \\
(1,3) \\
(3,4) \\
(1,2) \\
(2,3)\end{array}$ & $\begin{array}{l}.082 \\
.413 \\
.090 \\
.470 \\
.372 \\
.844\end{array}$ & $\begin{array}{r}.164 \\
.552 \\
.023 \\
.447 \\
1.010 \\
.846\end{array}$ & $\begin{array}{l}.041 \\
.368 \\
.072 \\
.525 \\
.461 \\
.782\end{array}$ & $\begin{array}{r}.082 \\
.491 \\
.019 \\
.499 \\
1.253 \\
.784\end{array}$ \\
\hline 60.0 & $\begin{array}{r}601.6 \\
1716.8 \\
2151.2 \\
3902.3 \\
4819.5 \\
5624.0\end{array}$ & $\begin{array}{l}(1,4) \\
(1,3) \\
(2,4) \\
(1,2) \\
(3,4) \\
(2,3)\end{array}$ & $\begin{array}{r}0.064 \\
.031 \\
.326 \\
.408 \\
.626 \\
.808\end{array}$ & $\begin{array}{r}0.189 \\
.113 \\
.470 \\
1.066 \\
0.490 \\
.873\end{array}$ & $\begin{array}{r}0.033 \\
.027 \\
.270 \\
.438 \\
.727 \\
.789\end{array}$ & $\begin{array}{r}0.097 \\
.099 \\
.389 \\
1.145 \\
0.569 \\
.852\end{array}$ \\
\hline 70.0 & $\begin{array}{r}653.5 \\
1298.4 \\
2460.2 \\
3603.5 \\
5173.8 \\
6039.1\end{array}$ & $\begin{array}{l}(1,4) \\
(1,3) \\
(2,4) \\
(1,2) \\
(2,3) \\
(3,4)\end{array}$ & $\begin{array}{l}.042 \\
.008 \\
.235 \\
.437 \\
.777 \\
.805\end{array}$ & $\begin{array}{r}.209 \\
.167 \\
.359 \\
1.080 \\
0.921 \\
.512\end{array}$ & $\begin{array}{l}.023 \\
.006 \\
.185 \\
.420 \\
.810 \\
.887\end{array}$ & $\begin{array}{r}.114 \\
.138 \\
.283 \\
1.038 \\
0.961 \\
.565\end{array}$ \\
\hline 80.0 & $\begin{array}{r}730.9 \\
1025.8 \\
2775.0 \\
3454.7 \\
4724.2 \\
6918.9\end{array}$ & $\begin{array}{l}(1,4) \\
(1,3) \\
(2,4) \\
(1,2) \\
(2,3) \\
(3,4)\end{array}$ & $\begin{array}{l}.021 \\
.000 \\
.110 \\
.454 \\
.774 \\
.953\end{array}$ & $\begin{array}{c}.233 \\
.179 \\
.174 \\
1.081 \\
1.001 \\
0.524\end{array}$ & $\begin{array}{l}.012 \\
.000 \\
.081 \\
.410 \\
.874 \\
.991\end{array}$ & $\begin{array}{r}.137 \\
.133 \\
.128 \\
.976 \\
1.130 \\
0.545\end{array}$ \\
\hline 90.0 & $\begin{array}{r}832.0 \\
864.8 \\
2936.7 \\
3409.4 \\
4507.8 \\
7237.3\end{array}$ & $\begin{array}{l}(1,4) \\
(1,3) \\
(2,4) \\
(1,2) \\
(2,3) \\
(3,4)\end{array}$ & $\begin{array}{r}.011 \\
.000 \\
.000 \\
.459 \\
.790 \\
1.010\end{array}$ & $\begin{array}{r}.439 \\
.000 \\
.000 \\
1.080 \\
1.062 \\
0.527\end{array}$ & $\begin{array}{r}.007 \\
.000 \\
.000 \\
.407 \\
.941 \\
1.027\end{array}$ & $\begin{array}{r}.273 \\
.000 \\
.000 \\
.956 \\
1.265 \\
0.536\end{array}$ \\
\hline
\end{tabular}


TABLE 4. The resonance magnetic field, the transition probability, T, and the integrated intensity, $\mathrm{U}$, of $\mathrm{Cr}^{3+}$ ion in ruby, for microwave frequency of $18.000 \mathrm{GHz}$

\begin{tabular}{|c|c|c|c|c|c|c|}
\hline ANGLE (Deg) & $H(\mathrm{Oe})$ & TRANS. & $T_{x^{\prime}}$ & $T_{y^{\prime}}$ & $U_{x^{\prime}}$ & $U_{y^{\prime}}$ \\
\hline 0.0 & $\begin{array}{r}1173.0 \\
2163.3 \\
2346.1 \\
5316.9 \\
6489.7 \\
10633.8\end{array}$ & $\begin{array}{l}(1,4) \\
(2,4) \\
(3,4) \\
(1,3) \\
(2,3) \\
(1,2)\end{array}$ & $\begin{array}{r}0.000 \\
.000 \\
.750 \\
.000 \\
1.000 \\
0.750\end{array}$ & $\begin{array}{r}0.000 \\
.000 \\
.750 \\
.000 \\
1.000 \\
0.750\end{array}$ & $\begin{array}{r}0.000 \\
.000 \\
.750 \\
.000 \\
1.000 \\
0.750\end{array}$ & $\begin{array}{r}0.000 \\
.000 \\
.750 \\
.000 \\
1.000 \\
0.750\end{array}$ \\
\hline 10.0 & $\begin{array}{r}1165.6 \\
2202.9 \\
2420.3 \\
5110.8 \\
6657.2 \\
10329.1\end{array}$ & $\begin{array}{l}(1,4) \\
(2,4) \\
(3,4) \\
(1,3) \\
(2,3) \\
(1,2)\end{array}$ & $\begin{array}{l}.012 \\
.054 \\
.725 \\
.158 \\
.957 \\
.718\end{array}$ & $\begin{array}{l}.013 \\
.055 \\
.731 \\
.154 \\
.948 \\
.756\end{array}$ & $\begin{array}{l}.006 \\
.020 \\
.728 \\
.085 \\
.913 \\
.731\end{array}$ & $\begin{array}{l}.006 \\
.020 \\
.734 \\
.083 \\
.904 \\
.770\end{array}$ \\
\hline 20.0 & $\begin{array}{l}1150.0 \\
2287.9 \\
2687.5 \\
4685.9 \\
6951.7 \\
9482.4\end{array}$ & $\begin{array}{l}(1,4) \\
(2,4) \\
(3,4) \\
(1,3) \\
(2,3) \\
(1,2)\end{array}$ & $\begin{array}{l}.037 \\
.240 \\
.645 \\
.257 \\
.921 \\
.636\end{array}$ & $\begin{array}{l}.041 \\
.250 \\
.662 \\
.231 \\
.896 \\
.774\end{array}$ & $\begin{array}{l}.018 \\
.108 \\
.641 \\
.146 \\
.844 \\
.681\end{array}$ & $\begin{array}{l}.019 \\
.113 \\
.658 \\
.132 \\
.821 \\
.828\end{array}$ \\
\hline 30.0 & $\begin{array}{l}1139.5 \\
2404.3 \\
3192.2 \\
4200.8 \\
7172.9 \\
8286.6\end{array}$ & $\begin{array}{l}(1,4) \\
(2,4) \\
(3,4) \\
(1,3) \\
(2,3) \\
(1,2)\end{array}$ & $\begin{array}{l}.057 \\
.315 \\
.582 \\
.253 \\
.910 \\
.542\end{array}$ & $\begin{array}{l}.069 \\
.342 \\
.601 \\
.183 \\
.876 \\
.809\end{array}$ & $\begin{array}{l}.027 \\
.164 \\
.603 \\
.145 \\
.825 \\
.617\end{array}$ & $\begin{array}{l}.032 \\
.178 \\
.623 \\
.105 \\
.794 \\
.921\end{array}$ \\
\hline 40.0 & $\begin{array}{l}1143.0 \\
2563.7 \\
3685.9 \\
3973.4 \\
7027.3 \\
7240.7\end{array}$ & $\begin{array}{l}(1,4) \\
(2,4) \\
(1,3) \\
(3,4) \\
(1,2) \\
(2,3)\end{array}$ & $\begin{array}{l}.065 \\
.319 \\
.188 \\
.572 \\
.476 \\
.902\end{array}$ & $\begin{array}{l}.093 \\
.366 \\
.080 \\
.569 \\
.864 \\
.873\end{array}$ & $\begin{array}{l}.030 \\
.181 \\
.109 \\
.629 \\
.564 \\
.825\end{array}$ & $\begin{array}{r}.042 \\
.208 \\
.046 \\
.627 \\
1.024 \\
.799\end{array}$ \\
\hline 50.0 & $\begin{array}{l}1166.0 \\
2773.8 \\
3146.1 \\
5032.2 \\
5981.4 \\
7127.9\end{array}$ & $\begin{array}{l}(1,4) \\
(2,4) \\
(1,3) \\
(3,4) \\
(1,2) \\
(2,3)\end{array}$ & $\begin{array}{l}.061 \\
.291 \\
.101 \\
.618 \\
.456 \\
.888\end{array}$ & $\begin{array}{l}.111 \\
.355 \\
.007 \\
.557 \\
.931 \\
.884\end{array}$ & $\begin{array}{l}.028 \\
.175 \\
.061 \\
.701 \\
.531 \\
.831\end{array}$ & $\begin{array}{r}.050 \\
.214 \\
.004 \\
.632 \\
1.083 \\
.827\end{array}$ \\
\hline 60.0 & $\begin{array}{l}1213.7 \\
2623.0 \\
3035.9 \\
5271.5 \\
6254.9 \\
6850.6\end{array}$ & $\begin{array}{l}(1,4) \\
(1,3) \\
(2,4) \\
(1,2) \\
(3,4) \\
(2,3)\end{array}$ & $\begin{array}{r}0.050 \\
.038 \\
.244 \\
.469 \\
.713 \\
.869\end{array}$ & $\begin{array}{r}0.125 \\
.008 \\
.315 \\
.985 \\
.556 \\
.908\end{array}$ & $\begin{array}{r}0.023 \\
.024 \\
.153 \\
.510 \\
.795 \\
.843\end{array}$ & $\begin{array}{r}0.057 \\
.005 \\
.197 \\
1.069 \\
0.619 \\
.881\end{array}$ \\
\hline 70.0 & $\begin{array}{l}1291.2 \\
2181.2 \\
3335.2 \\
4851.6 \\
6469.7 \\
7405.3\end{array}$ & $\begin{array}{l}(1,4) \\
(1,3) \\
(2,4) \\
(1,2) \\
(2,3) \\
(3,4)\end{array}$ & $\begin{array}{l}.034 \\
.009 \\
.172 \\
.490 \\
.857 \\
.831\end{array}$ & $\begin{array}{r}.136 \\
.037 \\
.232 \\
1.013 \\
.949 \\
.558\end{array}$ & $\begin{array}{l}.016 \\
.006 \\
.109 \\
.493 \\
.871 \\
.887\end{array}$ & $\begin{array}{r}.064 \\
.023 \\
.147 \\
1.021 \\
0.965 \\
.595\end{array}$ \\
\hline 80.0 & $\begin{array}{l}1404.3 \\
1849.6 \\
3613.3 \\
4634.0 \\
6106.4 \\
8221.7\end{array}$ & $\begin{array}{l}(1,4) \\
(1,3) \\
(2,4) \\
(1,2) \\
(2,3) \\
(3,4)\end{array}$ & $\begin{array}{l}.019 \\
.000 \\
.070 \\
.504 \\
.863 \\
.928\end{array}$ & $\begin{array}{c}.154 \\
.050 \\
.096 \\
1.024 \\
1.006 \\
0.560\end{array}$ & $\begin{array}{l}.009 \\
.000 \\
.043 \\
.483 \\
.923 \\
.952\end{array}$ & $\begin{array}{r}.076 \\
.030 \\
.059 \\
.982 \\
1.077 \\
0.574\end{array}$ \\
\hline 90.0 & $\begin{array}{l}1514.5 \\
1667.6 \\
3739.1 \\
4567.2 \\
5944.3 \\
8516.0\end{array}$ & $\begin{array}{l}(1,4) \\
(1,3) \\
(2,4) \\
(1,2) \\
(2,3) \\
(3,4)\end{array}$ & $\begin{array}{l}.013 \\
.000 \\
.000 \\
.509 \\
.873 \\
.966\end{array}$ & $\begin{array}{l}.230 \\
.000 \\
.000 \\
1.027 \\
1.040 \\
0.561\end{array}$ & $\begin{array}{l}.006 \\
.000 \\
.000 \\
.479 \\
.961 \\
.976\end{array}$ & $\begin{array}{r}.111 \\
.000 \\
.000 \\
.968 \\
1.145 \\
0.567\end{array}$ \\
\hline
\end{tabular}


TABLE 5. The resonance magnetic field, the transition probability, $\mathrm{T}$, and the integrated intensity, $\mathrm{U}$, of $\mathrm{Cr}^{3+}$ ion in ruby, for

\begin{tabular}{|c|c|c|c|c|c|c|}
\hline ANGLE (Deg) & $H(\mathrm{Oe})$ & TRANS. & $T_{x^{\prime}}$ & $T_{y^{\prime}}$ & $U_{x^{\prime}}$ & $U_{y^{\prime}}$ \\
\hline 0.0 & $\begin{array}{r}2254.7 \\
2884.4 \\
4509.4 \\
6398.4 \\
8653.3 \\
12796.9\end{array}$ & $\begin{array}{l}(1,4) \\
(2,4) \\
(3,4) \\
(1,3) \\
(2,3) \\
(1,2)\end{array}$ & $\begin{array}{r}0.000 \\
.000 \\
.750 \\
.000 \\
1.000 \\
0.750\end{array}$ & $\begin{array}{r}0.000 \\
.000 \\
.750 \\
.000 \\
1.000 \\
0.750\end{array}$ & $\begin{array}{r}0.000 \\
.000 \\
.750 \\
.000 \\
1.000 \\
0.750\end{array}$ & $\begin{array}{r}0.000 \\
.000 \\
.750 \\
.000 \\
1.000 \\
0.750\end{array}$ \\
\hline 10.0 & $\begin{array}{r}2220.3 \\
2963.3 \\
4609.4 \\
6245.6 \\
8759.3 \\
12521.5\end{array}$ & $\begin{array}{l}(1,4) \\
(2,4) \\
(3,4) \\
(1,3) \\
(2,3) \\
(1,2)\end{array}$ & $\begin{array}{l}.008 \\
.004 \\
.742 \\
.059 \\
.985 \\
.731\end{array}$ & $\begin{array}{l}.008 \\
.004 \\
.743 \\
.058 \\
.980 \\
.754\end{array}$ & $\begin{array}{l}.004 \\
.001 \\
.749 \\
.030 \\
.967 \\
.738\end{array}$ & $\begin{array}{l}.004 \\
.001 \\
.750 \\
.030 \\
.963 \\
.762\end{array}$ \\
\hline 20.0 & $\begin{array}{r}2155.9 \\
3153.7 \\
4928.1 \\
5871.8 \\
8983.4 \\
11755.9\end{array}$ & $\begin{array}{l}(1,4) \\
(2,4) \\
(3,4) \\
(1,3) \\
(2,3) \\
(1,2)\end{array}$ & $\begin{array}{l}.023 \\
.033 \\
.719 \\
.134 \\
.961 \\
.681\end{array}$ & $\begin{array}{l}.024 \\
.035 \\
.720 \\
.125 \\
.946 \\
.768\end{array}$ & $\begin{array}{l}.010 \\
.013 \\
.744 \\
.071 \\
.919 \\
.706\end{array}$ & $\begin{array}{l}.011 \\
.014 \\
.745 \\
.066 \\
.905 \\
.797\end{array}$ \\
\hline 30.0 & $\begin{array}{r}2104.7 \\
3396.1 \\
5397.0 \\
5502.0 \\
9177.7 \\
10664.6\end{array}$ & $\begin{array}{l}(1,4) \\
(2,4) \\
(1,3) \\
(3,4) \\
(2,3) \\
(1,2)\end{array}$ & $\begin{array}{l}.033 \\
.089 \\
.155 \\
.694 \\
.947 \\
.620\end{array}$ & $\begin{array}{l}.039 \\
.096 \\
.128 \\
.686 \\
.925 \\
.793\end{array}$ & $\begin{array}{l}.014 \\
.040 \\
.084 \\
.738 \\
.893 \\
.665\end{array}$ & $\begin{array}{l}.017 \\
.043 \\
.069 \\
.729 \\
.872 \\
.850\end{array}$ \\
\hline 40.0 & $\begin{array}{l}2083.2 \\
3667.6 \\
4883.2 \\
6350.6 \\
9252.0 \\
9475.6\end{array}$ & $\begin{array}{l}(1,4) \\
(2,4) \\
(1,3) \\
(3,4) \\
(2,3) \\
(1,2)\end{array}$ & $\begin{array}{l}.037 \\
.138 \\
.132 \\
.687 \\
.938 \\
.572\end{array}$ & $\begin{array}{l}.050 \\
.155 \\
.087 \\
.652 \\
.918 \\
.829\end{array}$ & $\begin{array}{l}.015 \\
.067 \\
.071 \\
.744 \\
.884 \\
.626\end{array}$ & $\begin{array}{l}.021 \\
.075 \\
.047 \\
.706 \\
.865 \\
.907\end{array}$ \\
\hline 50.0 & $\begin{array}{l}2097.3 \\
3965.2 \\
4359.0 \\
7428.7 \\
8407.2 \\
9172.9\end{array}$ & $\begin{array}{l}(1,4) \\
(2,4) \\
(1,3) \\
(3,4) \\
(1,2) \\
(2,3)\end{array}$ & $\begin{array}{l}.035 \\
.158 \\
.086 \\
.713 \\
.549 \\
.930\end{array}$ & $\begin{array}{l}.058 \\
.184 \\
.035 \\
.627 \\
.871 \\
.924\end{array}$ & $\begin{array}{l}.014 \\
.083 \\
.046 \\
.773 \\
.598 \\
.887\end{array}$ & $\begin{array}{l}.024 \\
.097 \\
.019 \\
.680 \\
.949 \\
.881\end{array}$ \\
\hline 60.0 & $\begin{array}{l}2149.6 \\
3853.9 \\
4285.9 \\
7590.8 \\
8606.0 \\
8953.1\end{array}$ & $\begin{array}{l}(1,4) \\
(1,3) \\
(2,4) \\
(1,2) \\
(3,4) \\
(2,3)\end{array}$ & $\begin{array}{r}0.028 \\
.043 \\
.145 \\
.547 \\
.769 \\
.923\end{array}$ & $\begin{array}{r}0.063 \\
.006 \\
.175 \\
.910 \\
.612 \\
.942\end{array}$ & $\begin{array}{r}0.012 \\
.023 \\
.079 \\
.579 \\
.820 \\
.900\end{array}$ & $\begin{array}{r}0.026 \\
.003 \\
.096 \\
.963 \\
.652 \\
.919\end{array}$ \\
\hline 70.0 & $\begin{array}{l}2241.4 \\
3407.8 \\
4606.6 \\
7049.8 \\
8651.4 \\
9676.8\end{array}$ & $\begin{array}{l}(1,4) \\
(1,3) \\
(2,4) \\
(1,2) \\
(2,3) \\
(3,4)\end{array}$ & $\begin{array}{l}.019 \\
.015 \\
.100 \\
.555 \\
.921 \\
.838\end{array}$ & $\begin{array}{l}.066 \\
.000 \\
.124 \\
.938 \\
.972 \\
.604\end{array}$ & $\begin{array}{l}.008 \\
.008 \\
.056 \\
.565 \\
.925 \\
.870\end{array}$ & $\begin{array}{l}.027 \\
.000 \\
.069 \\
.956 \\
.976 \\
.627\end{array}$ \\
\hline 80.0 & $\begin{array}{r}2365.0 \\
3060.5 \\
4867.2 \\
6749.0 \\
8376.5 \\
10425.8\end{array}$ & $\begin{array}{l}(1,4) \\
(1,3) \\
(2,4) \\
(1,2) \\
(2,3) \\
(3,4)\end{array}$ & $\begin{array}{l}.011 \\
.002 \\
.036 \\
.563 \\
.927 \\
.895\end{array}$ & $\begin{array}{r}.072 \\
.003 \\
.045 \\
.953 \\
1.005 \\
0.601\end{array}$ & $\begin{array}{l}.004 \\
.001 \\
.020 \\
.557 \\
.959 \\
.907\end{array}$ & $\begin{array}{r}.030 \\
.001 \\
.025 \\
.943 \\
1.040 \\
0.609\end{array}$ \\
\hline 90.0 & $\begin{array}{r}2448.8 \\
2900.8 \\
4972.3 \\
6653.3 \\
8261.2 \\
10695.3 \\
\end{array}$ & $\begin{array}{l}(1,4) \\
(1,3) \\
(2,4) \\
(1,2) \\
(2,3) \\
(3,4)\end{array}$ & $\begin{array}{l}.007 \\
.000 \\
.000 \\
.566 \\
.932 \\
.917 \\
\end{array}$ & $\begin{array}{r}.082 \\
.000 \\
.000 \\
.957 \\
1.022 \\
0.600\end{array}$ & $\begin{array}{l}.003 \\
.000 \\
.000 \\
.554 \\
.978 \\
.921 \\
\end{array}$ & $\begin{array}{r}.033 \\
.000 \\
.000 \\
.937 \\
1.073 \\
0.603 \\
\end{array}$ \\
\hline
\end{tabular}


TABLE 6. The resonance magnetic field, the transition probability, $\mathrm{T}$, and the integrated intensity, $\mathrm{U}$, of $\mathrm{Cr}^{3+}$ ion in ruby, for

\begin{tabular}{|c|c|c|c|c|c|c|}
\hline ANGLE (Deg) & $H(\mathrm{Oe})$ & TRANS. & $T_{x^{\prime}}$ & $T_{y^{\prime}}$ & $U_{x^{\prime}}$ & $U_{y^{\prime}}$ \\
\hline 0.0 & $\begin{array}{r}4206.2 \\
4237.5 \\
8381.3 \\
8475.6 \\
12619.1 \\
16762.7\end{array}$ & $\begin{array}{l}(1,4) \\
(2,4) \\
(1,3) \\
(3,4) \\
(2,3) \\
(1,2)\end{array}$ & $\begin{array}{r}0.000 \\
.000 \\
.000 \\
.750 \\
1.000 \\
0.750\end{array}$ & $\begin{array}{r}0.000 \\
.000 \\
.000 \\
.750 \\
1.000 \\
0.750\end{array}$ & $\begin{array}{r}0.000 \\
.000 \\
.000 \\
.750 \\
1.000 \\
0.750\end{array}$ & $\begin{array}{r}0.000 \\
.000 \\
.000 \\
.750 \\
1.000 \\
0.750\end{array}$ \\
\hline 10.0 & $\begin{array}{r}3991.8 \\
4507.4 \\
8259.8 \\
8603.0 \\
12684.6 \\
16515.4\end{array}$ & $\begin{array}{l}(1,4) \\
(2,4) \\
(1,3) \\
(3,4) \\
(2,3) \\
(1,2)\end{array}$ & $\begin{array}{l}.002 \\
.004 \\
.019 \\
.747 \\
.995 \\
.740\end{array}$ & $\begin{array}{l}.002 \\
.004 \\
.019 \\
.746 \\
.993 \\
.753\end{array}$ & $\begin{array}{l}.001 \\
.002 \\
.010 \\
.751 \\
.989 \\
.743\end{array}$ & $\begin{array}{l}.001 \\
.002 \\
.010 \\
.749 \\
.986 \\
.757\end{array}$ \\
\hline 20.0 & $\begin{array}{r}3820.3 \\
4839.1 \\
7933.6 \\
8990.7 \\
12835.9 \\
15822.8\end{array}$ & $\begin{array}{l}(1,4) \\
(2,4) \\
(1,3) \\
(3,4) \\
(2,3) \\
(1,2)\end{array}$ & $\begin{array}{l}.007 \\
.018 \\
.054 \\
.741 \\
.984 \\
.713\end{array}$ & $\begin{array}{l}.008 \\
.019 \\
.052 \\
.733 \\
.977 \\
.763\end{array}$ & $\begin{array}{l}.003 \\
.008 \\
.028 \\
.753 \\
.966 \\
.725\end{array}$ & $\begin{array}{l}.003 \\
.009 \\
.026 \\
.745 \\
.959 \\
.776\end{array}$ \\
\hline 30.0 & $\begin{array}{r}3706.6 \\
5206.1 \\
7479.0 \\
9644.5 \\
12982.9 \\
14819.3\end{array}$ & $\begin{array}{l}(1,4) \\
(2,4) \\
(1,3) \\
(3,4) \\
(2,3) \\
(1,2)\end{array}$ & $\begin{array}{l}.011 \\
.041 \\
.074 \\
.736 \\
.975 \\
.679\end{array}$ & $\begin{array}{l}.013 \\
.044 \\
.067 \\
.714 \\
.963 \\
.779\end{array}$ & $\begin{array}{l}.004 \\
.019 \\
.038 \\
.759 \\
.947 \\
.700\end{array}$ & $\begin{array}{l}.005 \\
.020 \\
.034 \\
.736 \\
.936 \\
.804\end{array}$ \\
\hline 40.0 & $\begin{array}{r}3649.0 \\
5595.2 \\
6962.2 \\
10545.4 \\
13049.8 \\
13689.5\end{array}$ & $\begin{array}{l}(1,4) \\
(2,4) \\
(1,3) \\
(3,4) \\
(2,3) \\
(1,2)\end{array}$ & $\begin{array}{l}.013 \\
.064 \\
.072 \\
.741 \\
.968 \\
.649\end{array}$ & $\begin{array}{l}.017 \\
.070 \\
.058 \\
.692 \\
.957 \\
.802\end{array}$ & $\begin{array}{l}.005 \\
.031 \\
.037 \\
.770 \\
.938 \\
.675\end{array}$ & $\begin{array}{l}.006 \\
.034 \\
.030 \\
.720 \\
.927 \\
.835\end{array}$ \\
\hline 50.0 & $\begin{array}{r}3644.7 \\
5994.6 \\
6429.7 \\
11624.5 \\
12613.3 \\
12999.0\end{array}$ & $\begin{array}{l}(1,4) \\
(2,4) \\
(1,3) \\
(3,4) \\
(1,2) \\
(2,3)\end{array}$ & $\begin{array}{l}.013 \\
.075 \\
.055 \\
.759 \\
.629 \\
.964\end{array}$ & $\begin{array}{l}.020 \\
.085 \\
.037 \\
.673 \\
.827 \\
.960\end{array}$ & $\begin{array}{l}.005 \\
.038 \\
.028 \\
.790 \\
.655 \\
.938\end{array}$ & $\begin{array}{l}.007 \\
.043 \\
.019 \\
.700 \\
.861 \\
.934\end{array}$ \\
\hline 60.0 & $\begin{array}{r}3690.4 \\
5919.4 \\
6386.0 \\
11722.2 \\
12750.0 \\
12840.8\end{array}$ & $\begin{array}{l}(1,4) \\
(1,3) \\
(2,4) \\
(1,2) \\
(3,4) \\
(2,3)\end{array}$ & $\begin{array}{r}0.010 \\
.033 \\
.069 \\
.621 \\
.791 \\
.962\end{array}$ & $\begin{array}{r}0.021 \\
.017 \\
.080 \\
.852 \\
.658 \\
.970\end{array}$ & $\begin{array}{r}0.004 \\
.017 \\
.036 \\
.639 \\
.815 \\
.947\end{array}$ & $\begin{array}{r}0.008 \\
.009 \\
.041 \\
.877 \\
.678 \\
.956\end{array}$ \\
\hline 70.0 & $\begin{array}{r}3777.3 \\
5472.2 \\
6736.1 \\
11080.1 \\
12624.0 \\
13744.6\end{array}$ & $\begin{array}{l}(1,4) \\
(1,3) \\
(2,4) \\
(1,2) \\
(2,3) \\
(3,4)\end{array}$ & $\begin{array}{l}.006 \\
.014 \\
.045 \\
.621 \\
.963 \\
.827\end{array}$ & $\begin{array}{l}.020 \\
.005 \\
.053 \\
.871 \\
.987 \\
.648\end{array}$ & $\begin{array}{l}.002 \\
.007 \\
.024 \\
.629 \\
.964 \\
.842\end{array}$ & $\begin{array}{l}.008 \\
.003 \\
.028 \\
.883 \\
.987 \\
.660\end{array}$ \\
\hline 80.0 & $\begin{array}{r}3879.1 \\
5142.6 \\
6989.7 \\
10700.2 \\
12433.6 \\
14429.7\end{array}$ & $\begin{array}{l}(1,4) \\
(1,3) \\
(2,4) \\
(1,2) \\
(2,3) \\
(3,4)\end{array}$ & $\begin{array}{l}.003 \\
.003 \\
.015 \\
.623 \\
.967 \\
.856\end{array}$ & $\begin{array}{r}.019 \\
.001 \\
.018 \\
.884 \\
1.003 \\
0.643\end{array}$ & $\begin{array}{l}.001 \\
.002 \\
.008 \\
.622 \\
.981 \\
.862\end{array}$ & $\begin{array}{r}.007 \\
.000 \\
.009 \\
.883 \\
1.018 \\
0.647\end{array}$ \\
\hline 90.0 & $\begin{array}{r}3930.9 \\
5012.7 \\
7084.2 \\
10575.2 \\
12356.9 \\
14673.8\end{array}$ & $\begin{array}{l}(1,4) \\
(1,3) \\
(2,4) \\
(1,2) \\
(2,3) \\
(3,4)\end{array}$ & $\begin{array}{l}.002 \\
.000 \\
.000 \\
.624 \\
.969 \\
.867\end{array}$ & $\begin{array}{r}.019 \\
.000 \\
.000 \\
.888 \\
1.010 \\
0.641\end{array}$ & $\begin{array}{l}.001 \\
.000 \\
.000 \\
.620 \\
.990 \\
.869\end{array}$ & $\begin{array}{l}.007 \\
.000 \\
.000 \\
.883 \\
1.032 \\
0.642\end{array}$ \\
\hline
\end{tabular}


TABLE 7. The resonance magnetic field, the transition probability, $\mathrm{T}$, and the integrated intensity, $\mathrm{U}$, of $\mathrm{Cr}^{3+}$ ion in ruby, for

\begin{tabular}{|c|c|c|c|c|c|c|}
\hline ANGLE (Deg.) & $H(\mathrm{Oe})$ & TRANS. & $T_{x^{\prime}}$ & $T_{y^{\prime}}$ & $U_{x^{\prime}}$ & $U_{u^{\prime}}$ \\
\hline 0.0 & $\begin{array}{r}6009.0 \\
6941.9 \\
11085.4 \\
13883.8 \\
18027.3 \\
22170.9\end{array}$ & $\begin{array}{l}(1,4) \\
(2,4) \\
(1,3) \\
(3,4) \\
(2,3) \\
(1,2)\end{array}$ & $\begin{array}{r}.000 \\
.000 \\
.000 \\
.750 \\
1.000 \\
0.750\end{array}$ & $\begin{array}{r}.000 \\
.000 \\
.000 \\
.750 \\
1.000 \\
0.750\end{array}$ & $\begin{array}{l}.000 \\
.000 \\
.000 \\
.750 \\
1.000 \\
0.750\end{array}$ & $\begin{array}{r}.000 \\
.000 \\
.000 \\
.750 \\
1.000 \\
0.750\end{array}$ \\
\hline 10.0 & $\begin{array}{r}5938.0 \\
7076.4 \\
10977.5 \\
14029.3 \\
18070.3 \\
21941.4\end{array}$ & $\begin{array}{l}(1,4) \\
(2,4) \\
(1,3) \\
(3,4) \\
(2,3) \\
(1,2)\end{array}$ & $\begin{array}{l}.000 \\
.003 \\
.007 \\
.749 \\
.998 \\
.744\end{array}$ & $\begin{array}{r}.000 \\
.003 \\
.007 \\
.747 \\
.997 \\
.752\end{array}$ & $\begin{array}{l}.000 \\
.002 \\
.004 \\
.751 \\
.995 \\
.746\end{array}$ & $\begin{array}{l}.000 \\
.002 \\
.004 \\
.749 \\
.994 \\
.754\end{array}$ \\
\hline 20.0 & $\begin{array}{r}5803.7 \\
7396.5 \\
10676.8 \\
14462.4 \\
18175.8 \\
21295.4\end{array}$ & $\begin{array}{l}(1,4) \\
(2,4) \\
(1,3) \\
(3,4) \\
(2,3) \\
(1,2)\end{array}$ & $\begin{array}{l}.001 \\
.011 \\
.023 \\
.749 \\
.993 \\
.729\end{array}$ & $\begin{array}{l}.001 \\
.012 \\
.022 \\
.739 \\
.989 \\
.759\end{array}$ & $\begin{array}{l}.000 \\
.006 \\
.012 \\
.755 \\
.984 \\
.735\end{array}$ & $\begin{array}{l}.001 \\
.006 \\
.011 \\
.745 \\
.981 \\
.766\end{array}$ \\
\hline 30.0 & $\begin{array}{r}5689.2 \\
7806.2 \\
10237.8 \\
15168.0 \\
18282.7 \\
20346.7\end{array}$ & $\begin{array}{l}(1,4) \\
(2,4) \\
(1,3) \\
(3,4) \\
(2,3) \\
(1,2)\end{array}$ & $\begin{array}{l}.003 \\
.023 \\
.035 \\
.750 \\
.988 \\
.708\end{array}$ & $\begin{array}{l}.003 \\
.024 \\
.033 \\
.726 \\
.982 \\
.771\end{array}$ & $\begin{array}{l}.001 \\
.011 \\
.018 \\
.761 \\
.974 \\
.719\end{array}$ & $\begin{array}{l}.001 \\
.012 \\
.017 \\
.737 \\
.968 \\
.782\end{array}$ \\
\hline 40.0 & $\begin{array}{r}5622.1 \\
8257.3 \\
9721.2 \\
16103.5 \\
18335.0 \\
19252.0\end{array}$ & $\begin{array}{l}(1,4) \\
(2,4) \\
(1,3) \\
(3,4) \\
(2,3) \\
(1,2)\end{array}$ & $\begin{array}{l}.004 \\
.033 \\
.038 \\
.757 \\
.984 \\
.689\end{array}$ & $\begin{array}{l}.005 \\
.036 \\
.033 \\
.712 \\
.978 \\
.786\end{array}$ & $\begin{array}{l}.001 \\
.016 \\
.019 \\
.772 \\
.968 \\
.703\end{array}$ & $\begin{array}{l}.002 \\
.018 \\
.017 \\
.726 \\
.962 \\
.801\end{array}$ \\
\hline 50.0 & $\begin{array}{r}5608.2 \\
8717.3 \\
9180.2 \\
17182.6 \\
18173.8 \\
18301.8\end{array}$ & $\begin{array}{l}(1,4) \\
(2,4) \\
(1,3) \\
(3,4) \\
(1,2) \\
(2,3)\end{array}$ & $\begin{array}{l}.004 \\
.038 \\
.031 \\
.771 \\
.674 \\
.981\end{array}$ & $\begin{array}{l}.006 \\
.042 \\
.025 \\
.698 \\
.802 \\
.979\end{array}$ & $\begin{array}{l}.001 \\
.019 \\
.016 \\
.786 \\
.688 \\
.967\end{array}$ & $\begin{array}{l}.002 \\
.021 \\
.013 \\
.711 \\
.818 \\
.965\end{array}$ \\
\hline 60.0 & $\begin{array}{r}5643.8 \\
8663.6 \\
9153.3 \\
17242.2 \\
18187.5 \\
18274.4\end{array}$ & $\begin{array}{l}(1,4) \\
(1,3) \\
(2,4) \\
(1,2) \\
(2,3) \\
(3,4)\end{array}$ & $\begin{array}{r}0.003 \\
.021 \\
.034 \\
.666 \\
.981 \\
.790\end{array}$ & $\begin{array}{r}0.006 \\
.015 \\
.038 \\
.819 \\
.985 \\
.686\end{array}$ & $\begin{array}{r}0.001 \\
.010 \\
.017 \\
.676 \\
.973 \\
.802\end{array}$ & $\begin{array}{r}0.002 \\
.008 \\
.019 \\
.830 \\
.977 \\
.697\end{array}$ \\
\hline 70.0 & $\begin{array}{r}5714.4 \\
8220.7 \\
9523.4 \\
16541.0 \\
18031.8 \\
19218.7\end{array}$ & $\begin{array}{l}(1,4) \\
(1,3) \\
(2,4) \\
(1,2) \\
(2,3) \\
(3,4)\end{array}$ & $\begin{array}{l}.002 \\
.010 \\
.021 \\
.663 \\
.982 \\
.811\end{array}$ & $\begin{array}{l}.006 \\
.006 \\
.024 \\
.832 \\
.994 \\
.678\end{array}$ & $\begin{array}{l}.001 \\
.005 \\
.011 \\
.667 \\
.982 \\
.818\end{array}$ & $\begin{array}{l}.002 \\
.003 \\
.012 \\
.838 \\
.993 \\
.683\end{array}$ \\
\hline 80.0 & $\begin{array}{r}5789.3 \\
7910.6 \\
9777.1 \\
16110.8 \\
17897.9 \\
19861.3\end{array}$ & $\begin{array}{l}(1,4) \\
(1,3) \\
(2,4) \\
(1,2) \\
(2,3) \\
(3,4)\end{array}$ & $\begin{array}{l}.001 \\
.003 \\
.007 \\
.662 \\
.984 \\
.827\end{array}$ & $\begin{array}{r}.005 \\
.001 \\
.008 \\
.841 \\
1.002 \\
0.673\end{array}$ & $\begin{array}{l}.000 \\
.001 \\
.003 \\
.662 \\
.991 \\
.830\end{array}$ & $\begin{array}{r}.002 \\
.001 \\
.004 \\
.841 \\
1.009 \\
0.675\end{array}$ \\
\hline 90.0 & $\begin{array}{r}5823.2 \\
7796.4 \\
9868.2 \\
15966.8 \\
17844.7 \\
20088.9 \\
\end{array}$ & $\begin{array}{l}(1,4) \\
(1,3) \\
(2,4) \\
(1,2) \\
(2,3) \\
(3,4)\end{array}$ & $\begin{array}{l}.000 \\
.000 \\
.000 \\
.662 \\
.985 \\
.833\end{array}$ & $\begin{array}{r}.005 \\
.000 \\
.000 \\
.844 \\
1.005 \\
0.671\end{array}$ & $\begin{array}{l}.000 \\
.000 \\
.000 \\
.661 \\
.995 \\
.834\end{array}$ & $\begin{array}{r}.002 \\
.000 \\
.000 \\
.842 \\
1.015 \\
0.671\end{array}$ \\
\hline
\end{tabular}




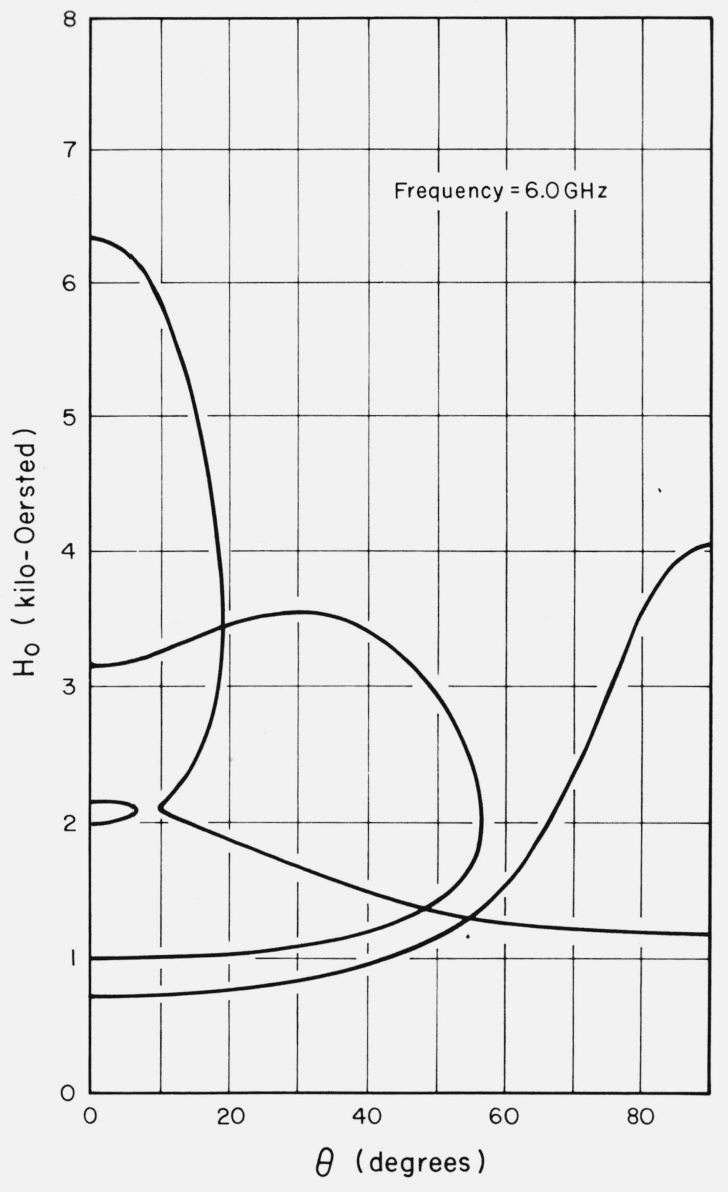

Figure 1. Paramagnetic resonance spectrum of $\mathrm{Cr}^{3+}$ in ruby at transition frequency of $6.000 \mathrm{GHz}$.

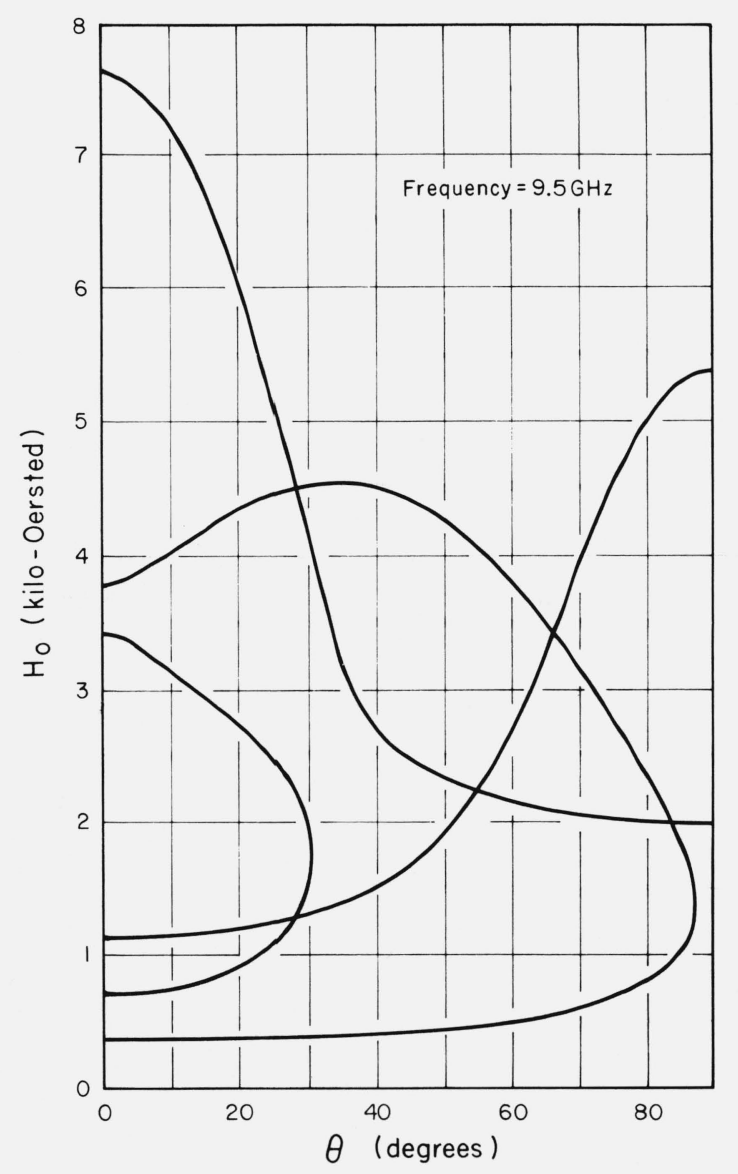




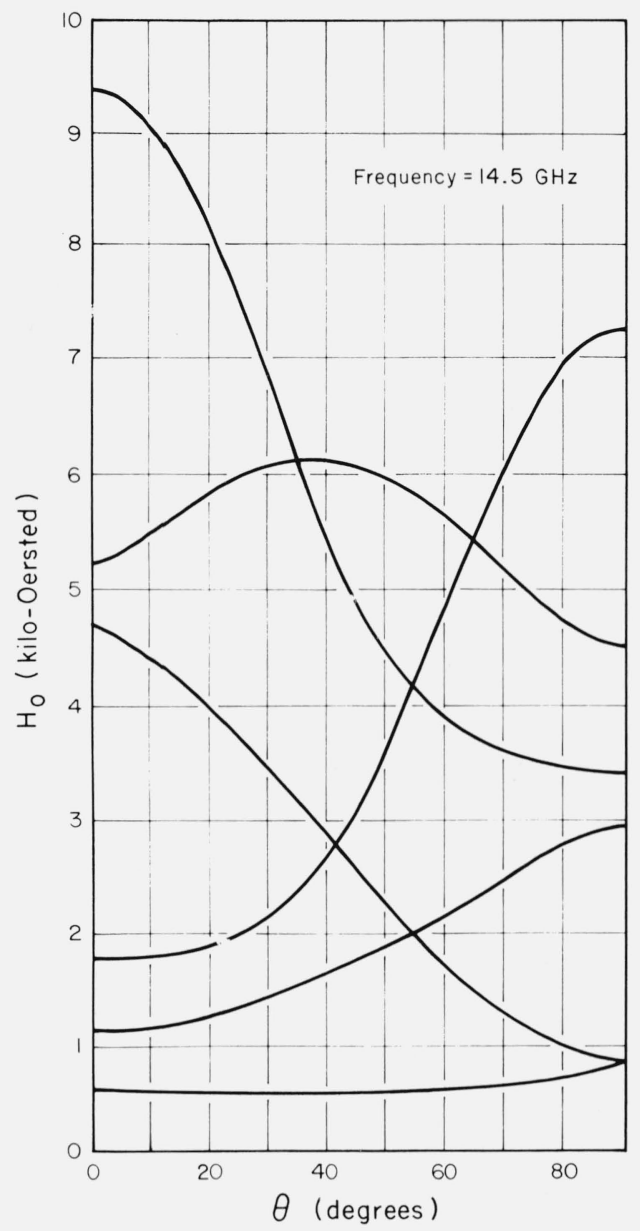

Figure 3. Resonance spectrum at $14.500 \mathrm{GHz}$.

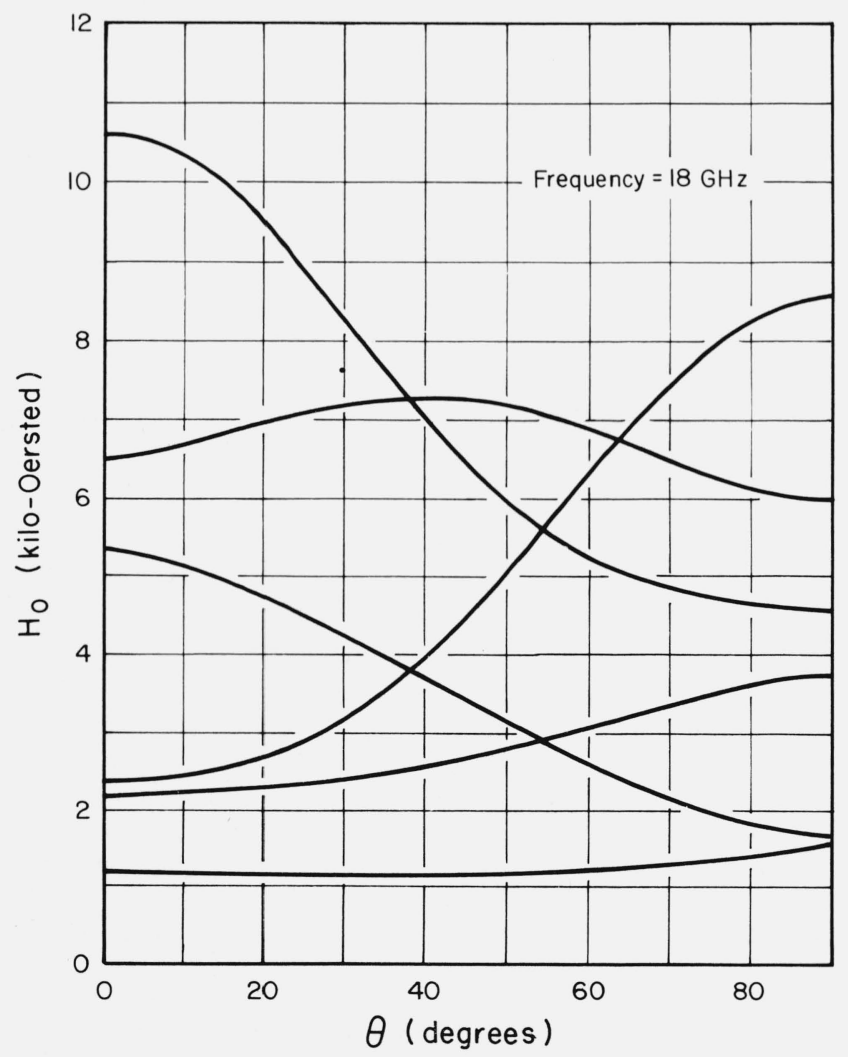




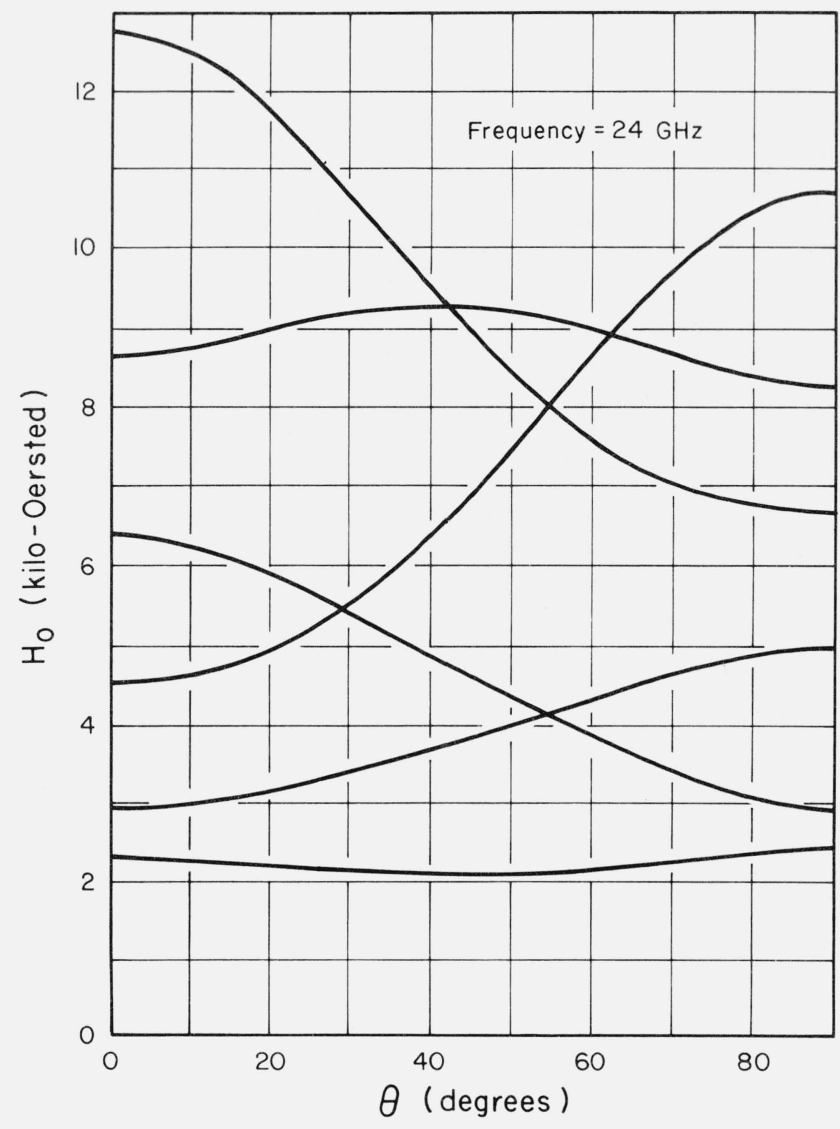

FIGURE 6. Resonance spectrum at $35.000 \mathrm{GHz}$.

Figure 5. Resonance spectrum at $24.000 \mathrm{GHz}$.

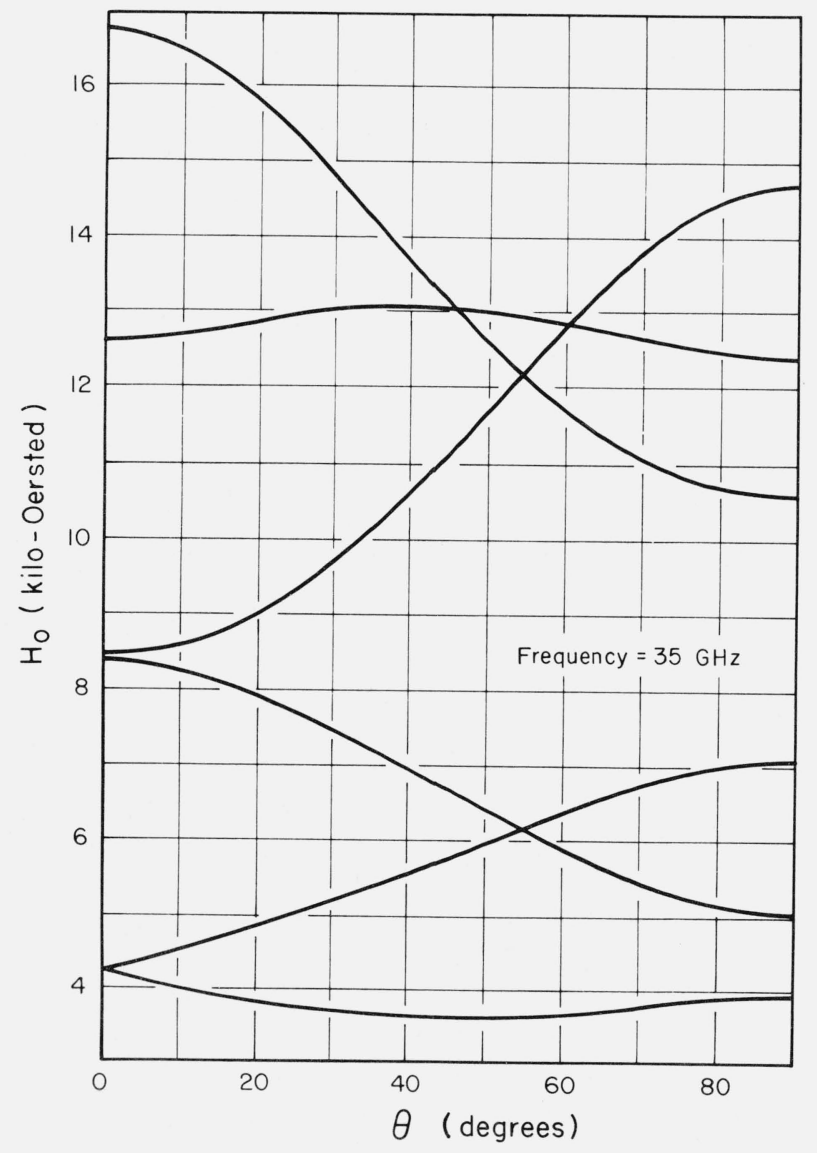




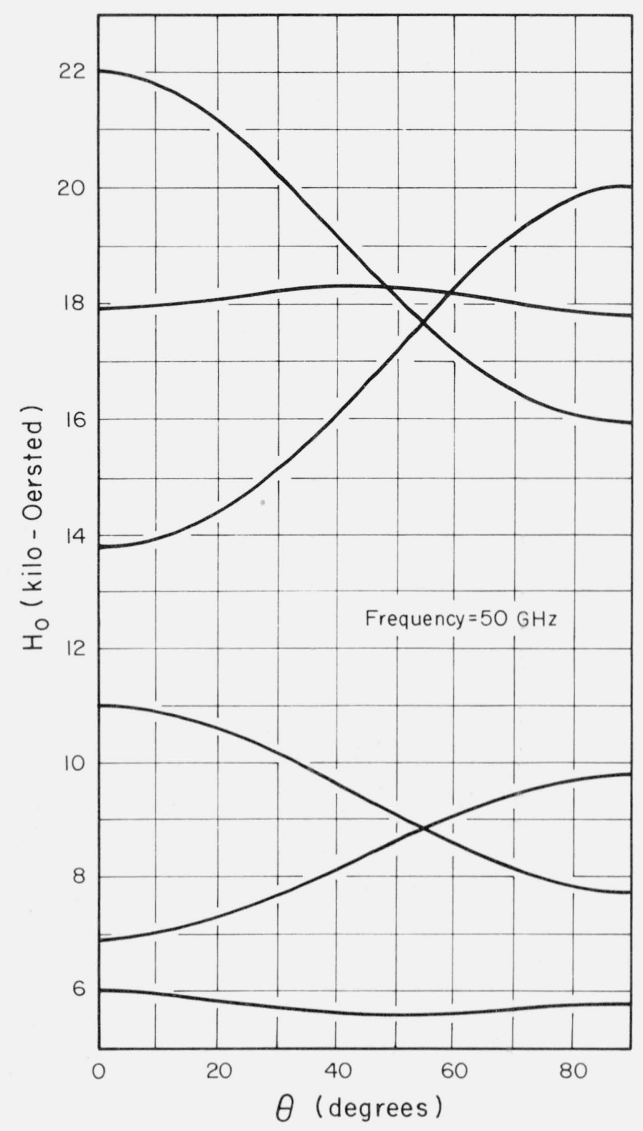

Figure 7. Resonance spectrum at $50.000 \mathrm{GHz}$.

The resonance absorption is detected by measuring absolutely, or more usually relatively, the change of cavity $Q$ as the static field $H_{0}$ is slowly swept through the region of resonance. The quality factor $Q$ of the cavity with a small sample present is given by

$$
\begin{aligned}
\frac{1}{Q} & =\frac{\text { Average Power Dissipated }}{\omega \cdot \text { Average Energy Stored }} \\
& =\frac{P_{\text {cav }}+P_{a}}{\frac{\omega}{8 \pi} \frac{1}{2} \int_{\text {cav }}\left[\left|H_{1}\right|^{2}+\left|E_{1}\right|^{2}\right] d v}
\end{aligned}
$$

where $P_{\text {cav }}$ is the power absorption rate of the cavity without the sample, $P_{a}$ is the power absorption rate of the sample as given by eq (2.15), and $E_{1}$ and $H_{1}$ are the values of the microwave fields throughout the cavity. We have assumed that the sample is sufficiently small that the losses are additive and that the microwave field in the cavity is not significantly changed by the presence of the sample. Then, since the magnetic and electric stored energies are equal we obtain

$$
\frac{1}{Q}=\frac{1}{Q_{0}}+\frac{P_{a}}{\frac{\omega}{8 \pi} \int_{\mathrm{cav}}\left|H_{1}\right|^{2} d v}
$$


where we identify

$$
\frac{1}{Q_{0}}=\frac{P_{\mathrm{cav}}}{\frac{\omega}{8 \pi} \int\left|H_{1}\right|^{2} d v}
$$

as the reciprocal of the quality factor for the empty cavity.

The energy absorption rate of the sample which is detected in the experiment may be expressed in terms of the imaginary part of the susceptibility $\chi^{\prime \prime}$ by the formula [14]

$$
P_{a}=(1 / 2) \omega H_{1}^{2} V_{s} \chi^{\prime \prime},
$$

where $V_{s}$ is the sample volume. We define the filling factor $\eta$, a dimensionless quantity by

$$
\eta=\frac{H_{1}^{2} V_{s}}{\int_{\text {cav }}\left|H_{1}\right|^{2} d v} .
$$

Filling factors are tabulated for various cavity shapes and sample positions in the text of Poole [15]. Finally we obtain

$$
\frac{1}{Q}=\frac{1}{Q_{0}}+4 \pi \chi^{\prime \prime} \eta
$$

relating the $Q$ to the susceptibility. If $H_{0}$ is far from a resonance, the presence of the sample has little effect. In the vicinity of resonance, the susceptibility is measured through the variation in $Q$. The form of the susceptibility from eqs (2.15) and (2.35) is

$$
\chi^{\prime \prime}(\nu)=\frac{\pi N_{0} \nu \bar{g}^{2} \mu_{B}^{2}}{(2 S+1) k T V_{s}} \sum_{\alpha<\beta}\left|\left\langle\alpha\left|S_{l}\right| \beta\right\rangle\right|^{2} g_{\alpha \beta}(\nu),
$$

where the validity of eq (2.38) requires that the individually normalized line shapes $g_{\alpha \beta}(\nu)$ do not overlap. Application of a Kramers-Kronig relation [16] gives the real part of the static susceptibility $\chi^{\prime}(0)$

$$
\chi^{\prime}(0)=\frac{2}{\pi} \int_{0}^{\infty} \frac{\chi^{\prime \prime}(\nu)}{\nu} d \nu
$$

where $\chi(\nu)=\chi^{\prime}(\nu)+i \chi^{\prime \prime}(\nu)$. An exact evaluation of the matrix element sums is possible and the resultant expression is

$$
\chi^{\prime}(0)=\frac{N_{0}}{V_{s}} \frac{g^{2} \mu_{B}^{2} S(S+1)}{3 k T}
$$

as is expected from the known paramagnetic Curie law.

Equation (2.38) is deceptively simple. Since the rf frequency is held fixed, it is possible that certain $(\alpha, \beta)$ transitions will not be accessible at any value of field $H_{0}$. Also, certain transitions may be observed more than once in a sweep of the field $H_{0}$. For any single resonance that is observable, the form of $g(\boldsymbol{\nu})$ is obtained from the field-sweep shape $f\left(H_{0}\right)$ divided by the weighting factor, $|d \nu / d H|$.

\subsection{Comparison With a Standard Sample}

The principal intended use of the EPR ruby standard is the production of a standard EPR signal with which the signals from a test sample may be compared. From comparison of line intensities one would hope to obtain 
an estimate of the number of spins in the test sample. This section will describe procedures and use of the preceding analysis in several cases for which the comparison is possible.

In this publication, the integrated intensity of an EPR line will be defined by the process that follows. The resonance line is scanned by sweeping the magnetic field $\mathrm{H}_{0}$ through the line profile. Since field modulation is used, the EPR spectrometer records the derivative of the field-sweep line, $f^{\prime}\left(H_{\mathrm{o}}\right)$. A first integration, extended from beyond the absorption region to any point in the line profile gives the value of line shape function at that point $f\left(H_{0}\right)$. A second integration over the full width of the line shape function gives the area under the curve of $f\left(H_{0}\right)$, which we call the integrated intensity of the line, $I$. This quantity is proportional to the integral of the imaginary part of the susceptibility, $\chi^{\prime \prime}$. The ratio of the integrated intensity of a test sample resonance to that of a standard sample resonance is ideally independent of spectrometer conditions.

Let us consider the comparison of integrated intensities of two resonance lines which occur for different fields $H_{0}$. The resonances could arise from different species in one sample, or from spins of different samples placed in the cavity at the same time. One of the resonances to be considered is from a standard sample, the other from a test sample. We assume that both samples are subject to identical microwave field amplitudes. In the following, we shall let a zero subscript refer to quantities relevant to the "standard" resonance. Using eqs (2.35) and (2.38), we obtain the following expression for the ratio of the integrated field-sweep intensities of the two resonances:

$$
\frac{I}{I_{0}}=-\frac{\frac{N g^{2}}{2 S+1}\left|\left\langle\alpha\left|S_{l}\right| \beta\right\rangle\right|^{2} \frac{1}{\left|d \nu_{\alpha \beta} / d H\right|}}{\frac{N_{0} g_{0}^{-2}}{2 S_{0}+1}\left|\left\langle\alpha_{0}\left|S_{l}\right| \beta_{0}\right\rangle\right|^{2} \frac{1}{\left|d \nu_{\alpha_{0} \beta_{0}} / d H_{0}\right|}}
$$

where $(\alpha, \beta)$ denotes the transition of the unknown and $\left(\alpha_{0}, \beta_{0}\right)$ denotes the transition of the standard. If the standard is $\mathrm{Cr}^{3+}$ in ruby, $\mathrm{S}_{0}=3 / 2$ and the matrix element and derivative in the denominator may be obtained from the tabulated values of $U_{l}^{a_{0} \beta_{0}}$. Then we have

$$
\frac{I}{I_{0}}=\frac{\frac{N g^{2}}{2 S+1}\left|\left\langle\alpha\left|S_{l}\right| \beta\right\rangle\right|^{2} \frac{1}{\left|d \nu_{\alpha \beta} / d H\right|}}{\frac{N_{0} g_{0}^{2}}{4} U_{l}^{\alpha_{0} \beta_{0}}-\frac{h}{g_{0} \mu_{B}}}
$$

We now consider several practical examples in which the integrated intensity ratios of eq (2.42) assume a simpler form. These are as follows:

(1) Test sample has $S=1 / 2$; ruby standard oriented with $\theta=0^{\circ},(1 / 2,-1 / 2)$ transition; $x$ or $y$ polarization. In this case the intensity ratio becomes

$$
\frac{I}{I_{0}}=\frac{1}{2} \frac{N}{N_{0}} \frac{g}{g_{0}}
$$

For this configuration the ratio of the number of spins is easily determined. This is the simplest use of the EPR standard. This use requires knowledge of the $g$-factor of the paramagnetic species of the test sample, and of course, the resonance absorption lines must not overlap.

(2) Test sample has $S=1 / 2$; ruby standard oriented at angle $\theta_{0}, y^{\prime}$ polarization, transition $\left(\alpha_{0}, \beta_{0}\right)$. This is a case that would occur if the condition of (1) were met, but the resonance lines were found to overlap so that the two intensities could not be measured. Then the resonance of the standard could be shifted to a convenient value of field by positioning the standard with its $c$ axis at angle $\theta_{0}$ with respect to the field. The intensity ratio becomes

$$
\frac{I}{I_{0}}=\frac{N}{2 N_{0}} \frac{g}{g_{0}}-\frac{1}{U_{y^{\prime}}^{\alpha_{0} \beta_{0}}}
$$

The value of $U_{y}^{a^{\prime}} \beta_{0}$ for the appropriate frequency and orientation angle is obtained from the numerical tables. 


\section{Preparation of Samples}

Ruby, commercially produced by the Czochralski process, was used as the starting material. The nominal doping is 0.05 percent by weight, i.e., 0.05 percent of $\mathrm{Al}_{2} \mathrm{O}_{3}$ by weight is $\mathrm{Cr}_{2} \mathrm{O}_{3}$. This concentration is equivalent to $0.067 \mathrm{~mol}$ percent of $\mathrm{Cr}$ in $\mathrm{Al}_{2} \mathrm{O}_{3}$. The actual concentration in the boule is usually lower. From our measurement, the $\mathrm{Cr}^{3+}$ concentration is about ten percent lower than the nominal doping.

The color of the boule appears to be uniform. From the investigation of Blunt [17], there is a slight short range variation of concentration from point to point. However, the EPR samples are sufficiently large, that the average concentration is detected in our measurements.

The crystalline axis, which is also the trigonal axis of the local symmetry, is also a unique axis of the $\mathrm{Cr}^{3+}$ EPR spectrum. The wandering of the $c$ axis is in the order of $0.01^{\circ}$ [18] which is essentially zero for the practical purposes of EPR work.

A precision wafering machine was used to cut the boule. This machine had been carefully aligned before use. The boule was sliced into two types of thin slices; one type with the $c$ axis perpendicular to the surface and the other type with the $c$ axis parallel to the surface. The orientation was within $1^{\circ}$. Then the slice was mounted on a jig specially made for fine adjustment of small angles. The orientation was readjusted to within $0.1^{\circ}$. The determination of the orientation was by $x$-ray Laue back scattering photographs, using a commercially made x-ray diffractometer. The collimator of the x-ray camera was $57 \mathrm{~mm}$ long and had a 0.25 $\mathrm{mm}$ bore. The alignment of the camera had to be performed carefully because of the very small bore of the collimator. The exposure time usually was about four hours. The spots on the film were round and well defined when the alignment was correct. The spots from the same zone could be connected by a straight line. The intersection of several straight lines was at the center of the film if the orientation was correct. A deviation of $0.1^{\circ}$ could be detected. The surface of the slices was then corrected by grinding with a diamond embedded wheel and the thickness was adjusted as well. The slice was cut either into $1.5 \times 1.5 \times 0.5 \mathrm{~mm}$ squares with the $c$ axis perpendicular to the square surface, or into $0.5 \times 0.5 \times 4 \mathrm{~mm}$ bars with the $c$ axis parallel to the long edge. The first batch of standard reference samples contains 120 sets. Each set consists of one square sample and one bar-like sample.

The as-cut surface of ruby has a flat white appearance. The subsurface is heavily strained. To remove part of the strain, the samples were annealed in an argon filled, resistance heated furnace. The argon atmosphere is a design feature of the furnace, and it was not selected for the purpose of annealing the ruby. The samples were washed in diluted acid and in alcohol first, to remove contamination or traces of oil, then loaded into a tungsten rucible and separated into layers by tungsten sheets to avoid wetting. The annealing temperature was about $1800{ }^{\circ} \mathrm{C}$. When the temperature was reached, the heating was gradually reduced and the samples removed. Annealing at temperatures higher than $1800{ }^{\circ} \mathrm{C}$ may result in near melting of the samples due to occasional surges in the power supply or uneven thermal distribution in the oven. After annealing, the samples were heated to $1000{ }^{\circ} \mathrm{C}$ in a platinum crucible in air, overnight, to restore oxygen if there had been any loss during annealing in the argon atmosphere. Then the samples were placed in platinum wire baskets and etched in phosphoric acid at $500{ }^{\circ} \mathrm{C}$. The etching operation was brief. When the temperature reached 500 ${ }^{\circ} \mathrm{C}$, the sample was removed from the acid bath promptly to avoid the redeposit of dissolved ruby and then plunged into boiling water to remove excess acid. Removal of redeposits was difficult, and the most effective procedure was to etch them again. Repeated etching may deform the sample.

The finished surface is smooth and the entire sample is transparent. Examined under a microscope, the surface looks smooth and glazed, and sometimes patterns like flow lines may appear on the surface. The corners and edges are also rounded. Spot checks of orientation after treatment indicate the inaccuracy is less than $0.5^{\circ}$.

This elaborate treatment of samples is very important and essential for the SRM. The reasons will be discussed later. Reversing the order of annealing and etching sometimes led to cracking or contamination of the samples.

\section{The Spectrometer}

The spectrometer is of the conventional autodyne design as shown in the block diagram in figure 8 . The $\mathrm{X}$ band klystron is immersed in an oil bath for temperature stability. The K-band $(24.0 \mathrm{GHz})$ klystron, since it 


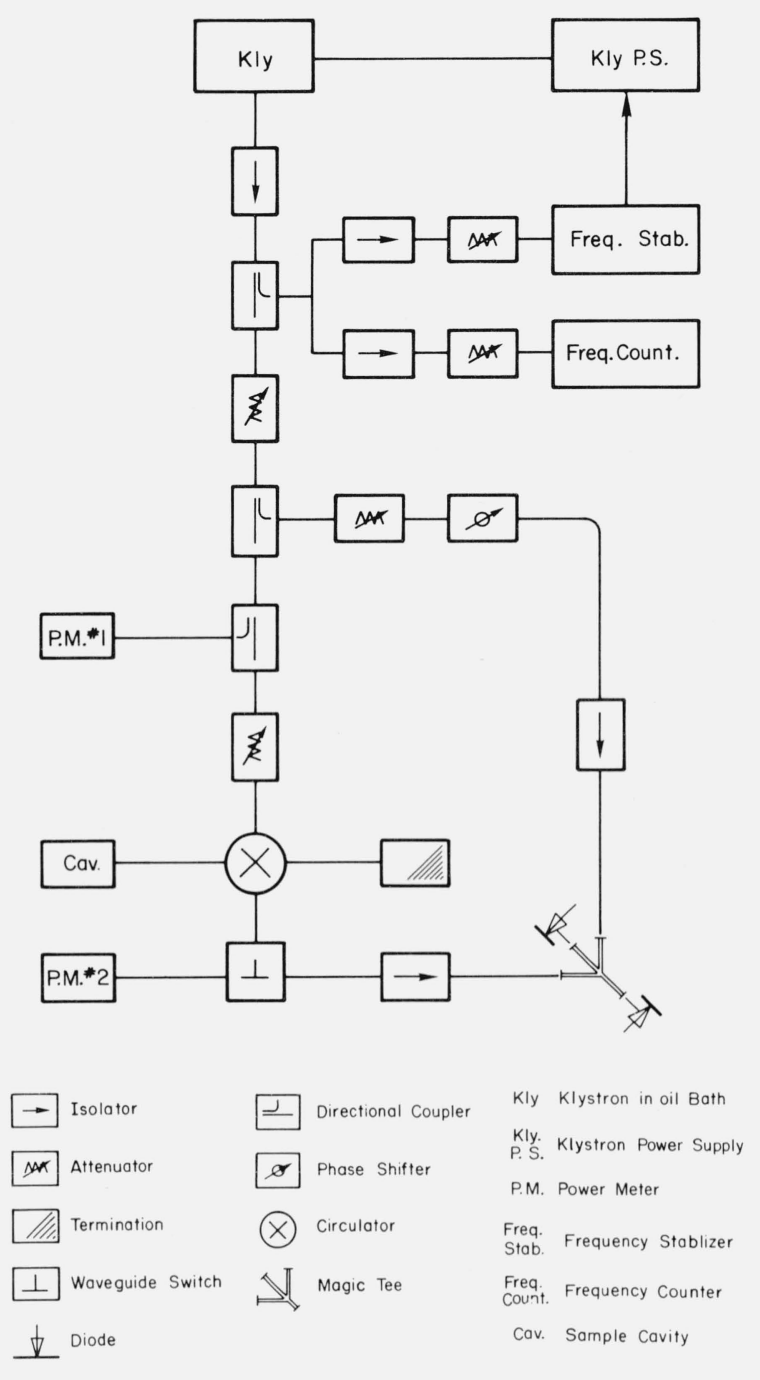

Figure 8. Block diagram of the EPR spectrometer.

has external cavity tuning, is mounted on a thick brass plate for dissipation of heat and stability of temperature. The microwave frequency is stabilized with a phase-locked stabilizer. The reference frequency is derived from an oscillator controlled by quartz plates inside a constant temperature oven. The microwave frequency can be stabilized to one part in $10^{7}$ or better. The reflected power from the sample cavity could be directed by the waveguide switch either to the power meter or to the bridge. The final mixing of the signal and the bucking power is accomplished at the magic tee. Two diodes of opposite polarities are used for detection. The signal is fed to the phase sensitive lock-in detector through a split coil audio frequency transformer to match the impedance and to provide the D.C. return for the diodes. The final output from the lock-in detector can be fed to a strip chart recorder or to a digital data acquisition system.

In the double diode detection system, the noise depends sharply on the autodyne phase setting which will aid the tuning of the bridge. The position of minimum noise is the optimum condition for balancing the bridge and at most times a good EPR absorption signal is secured. It is better to keep the signal power very low to maintain a "good" balance; hence, critical coupling of the sample cavity to the microwave system is essential.

We used several variable coupling schemes to obtain "critical" coupling. 
The first method is the cable coupling scheme [19] (fig. 9). The final connection to the cavity is through coaxial cable terminated in a loop. The loop is placed opposite the coupling iris of the cavity. The distance between the loop and the iris determined the coupling. This method is especially suitable for X-band or lower frequencies. The coaxial cable also has a higher attenuation than the waveguide so the cavity is essentially loosely coupled to the microwave system, and this is an additional advantage of this scheme.

The second method is Gordon's dielectric plunger scheme [20] (fig. 10). A segment of the waveguide immediately preceding the cavity is tapered to beyond cut off. A tapered dielectric plunger is inserted into the waveguide to bring this segment above cut off again. The plunger can be moved in the waveguide, and the distance between the plunger and the iris determines the coupling.
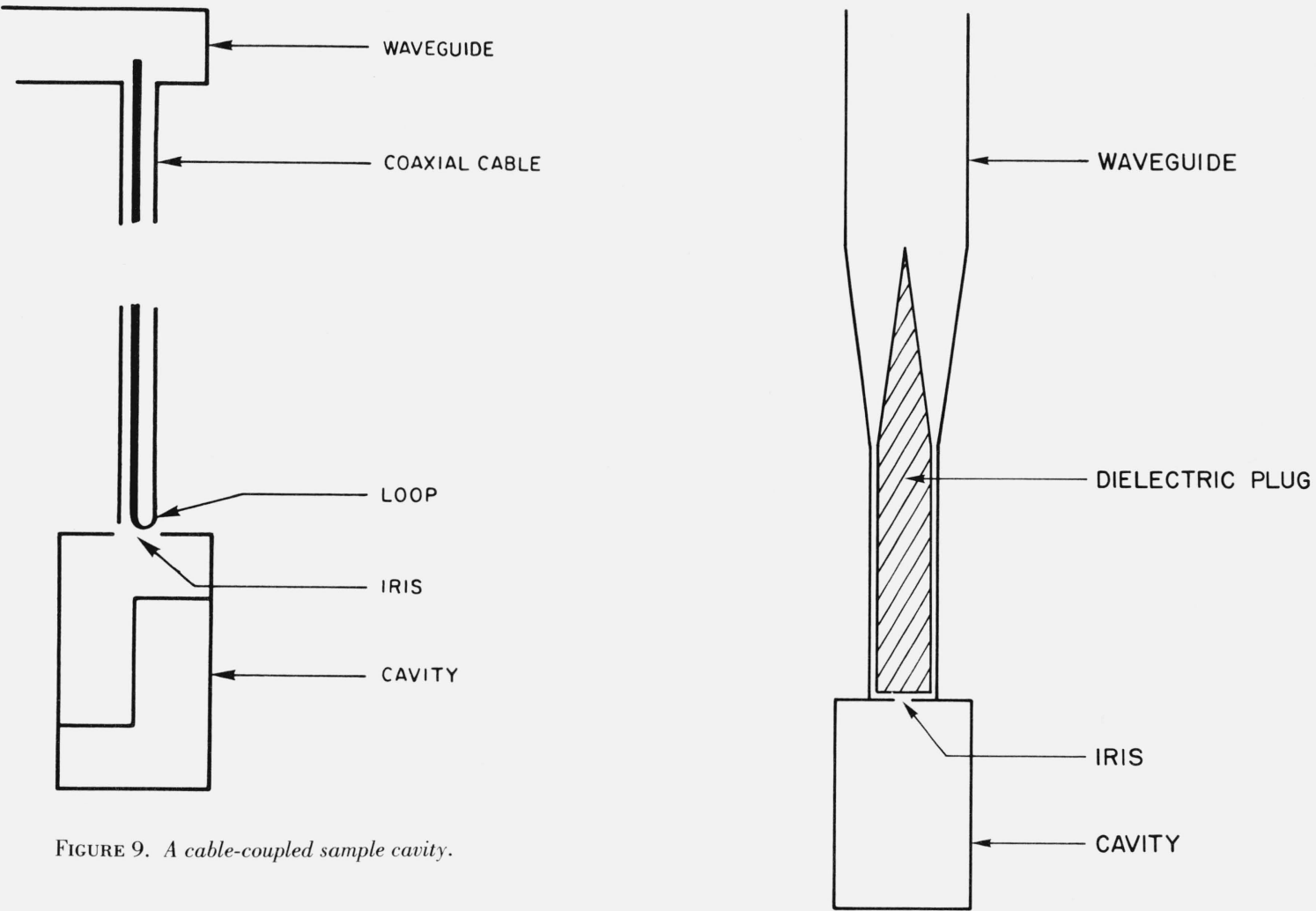

FIGURE 9. A cable-coupled sample cavity.

Figure 10. A dielectric plunger-coupled cavity.

The third method uses a capacitive adjustment (fig. 11). A dielectric screw with a metal (brass or copper) disc tip is inserted into the waveguide immediately above the iris parallel to the wider side of the wave guide. The metal disc need not contact the waveguide or the iris. The relative position of the metal disc and the iris determines the coupling.

By the above schemes, together with the highly stabilized frequency, the coupling can be adjusted to the equivalent of $50 \mathrm{~dB}$ attenuation or greater; i.e., the reflected power from the cavity is more than $50 \mathrm{~dB}$ below the input power. Practically, due to stray reflection of the components, and the characteristics of the microwave cavity, it is not possible to reduce the reflected power further. Usually, $30 \mathrm{~dB}$ attenuation is considered to be "critical" coupling.

In the X-band measurements, a rectangular $\mathrm{TE}_{102}$ cavity (fig. 9) is used. The cavity is sliced into two parts along the surface current lines for easy access to the interior of the cavity, and maximum visual observation, while mounting the sample. During an angular dependence study, while the magnet is rotated, the modulation 
field may experience different effective thickness and area of the cavity wall. Hence, the modulation amplitude at the sample will not be the same at various angular positions, and the signal intensity will vary accordingly. This variation had been observed.

For absolute measurements at $9.5 \mathrm{GHz}$ and all measurements at $24 \mathrm{GHz}, \mathrm{TE}_{011}$ cylindrical cavities are used.

The magnetic field was provided by a twelve inch electromagnet. This magnet can be rotated about its vertical axis, i.e., the magnetic field can be directed at any direction in the horizontal plane. The angular position can be measured to $0.1^{\circ}$. The dewar flask, the wave guide, and the microwave cavity are placed along the vertical axis, hence the angular dependence study can be easily performed.
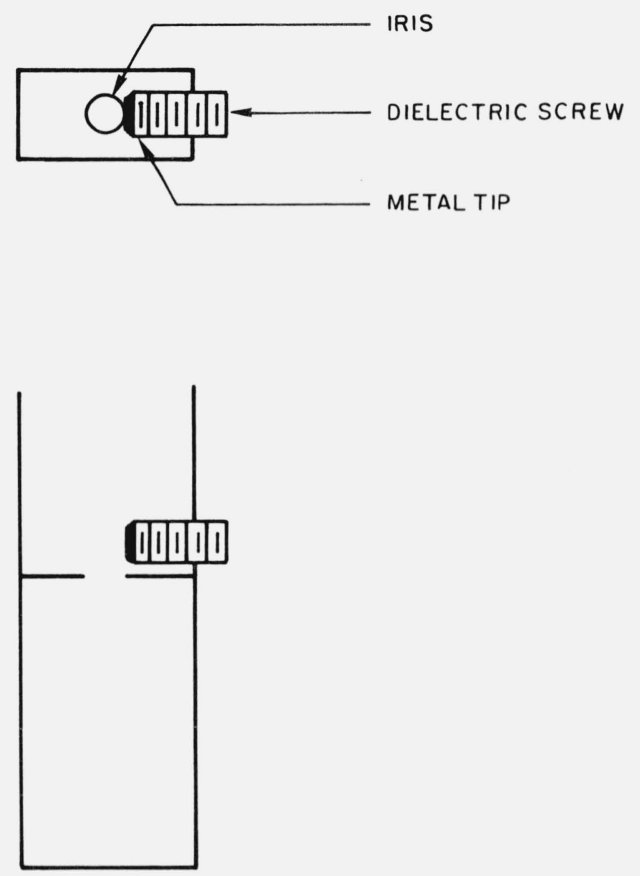

FigURE 11. A capacitive-coupled sample cavity.

\section{Results}

\subsection{The Hamiltonian Parameters}

For $\theta=0^{\circ}$ the secular equation can be factored and the transition frequency can be expressed in analytical form:

$$
\begin{array}{ll}
h \nu=+2 D+g_{\|} \mu_{B} H & \text { for the }(3 / 2,1 / 2) \text { transition. } \\
h \nu=g_{\|} \mu_{B} H & \text { for the }(1 / 2,-1 / 2) \text { transition } \\
h \nu=-2 D+g_{\|} \mu_{B} H & \text { for the }(-1 / 2,-3 / 2) \text { transition. }
\end{array}
$$

It follows from (5.3) that if the microwave frequency is less than the zero field splitting, $-2 D / h$, the $(-1 / 2,-3 / 2)$ transition cannot be observed. For $6.0 \mathrm{GHz}$, the $(1 / 2,-1 / 2)$ transition appears as the $(1,3)$ transition at $2163.3 \mathrm{Oe}$; the $(3 / 2,1 / 2)$ transition, as the $(1,2)$ transition at $6306.6 \mathrm{Oe}$, and again as the $(1,3)$ transition at 1980.5 Oe. For $9.5 \mathrm{GHz}$, the $(1 / 2,-1 / 2)$ transition appears as the $(1,3)$ transition at 3425.0 Oe; the $(3 / 2,1 / 2)$ transition appears as the $(1,2)$ transition at $7568.9 \mathrm{Oe}$, and the $(1,3)$, at 718.8 Oe. At 14.5 
$\mathrm{GHz}$, the $(3 / 2,1 / 2)$ transition appears as the $(1,2)$ transition; the $(1 / 2,-1 / 2)$, as the $(2,3)$; and the $(-1 / 2,-3 /$ $2)$, as the $(2,4)$. For $18.0 \mathrm{GHz}$ or higher frequency, the $(3 / 2,1 / 2),(1 / 2,-1 / 2)$, and $(-1 / 2,-3 / 2)$ transitions are respectively the $(1,2),(2,3)$, and $(3,4)$ transitions. Both the microwave frequency and the magnetic field can be measured, so $g_{\|}$and $2 D$ can be evaluated from the experimental data.

At $90^{\circ}$, the secular equation can be factored also, but the expressions for the transition frequency are somewhat more complicated than those at $0^{\circ}$. They are

$$
\begin{aligned}
& h \nu=g_{\perp} \mu_{B} H+\left(D^{2}-D g_{\perp} \mu_{B} H+g_{\perp}^{2} \mu_{B}^{2} H^{2}\right)^{1 / 2}-\left(D^{2}+D g_{\perp} \mu_{B} H+g_{\perp}^{2} \mu_{B}^{2} H^{2}\right)^{1 / 2} \\
& \text { for the }(1,2) \text { transition } \\
& h \nu=-g_{\perp} \mu_{B} H+\left(D^{2}-D g_{\perp} \mu_{B} H+g_{\perp}^{2} \mu_{B}^{2} H^{2}\right)^{1 / 2}+\left(D^{2}+D g_{\perp} \mu_{B} H+g_{\perp}^{2} \mu_{B}^{2} H^{2}\right)^{1 / 2} \\
& \text { for the }(2,3) \text { transition } \\
& h \nu=g_{\perp} \mu_{B} H-\left(D^{2}-D g_{\perp} \mu_{B} H+g_{\perp}^{2} \mu_{B}^{2} H^{2}\right)^{1 / 2}+\left(D^{2}+D g_{\perp} \mu_{B} H+g_{\perp}^{2} \mu_{B}^{2} H^{2}\right)^{1 / 2}
\end{aligned}
$$

for the $(3,4)$ transition.

The value for $g_{\perp}$ can be obtained by fitting the experimental data to these equations and using the previously determined values of $D$.

From our measurements at $9.5 \mathrm{GHz}$ and at $24 \mathrm{GHz}$ we obtained,

$$
\begin{aligned}
g_{\|} & =1.9817 \pm 0.0004 \\
g_{\perp} & =1.9819 \pm 0.0006 \\
\frac{2 D}{h} & =-11.493 \pm 0.004 \mathrm{GHz}
\end{aligned}
$$

where the precision indices are probable errors. These results are in reasonable agreement with published values [5, 8].

The errors arise from four independent contributions. They are as follows: (1) Error in the measurement of the magnetic field from the line width of the proton resonance probe was \pm 0.1 Oe; (2) Magnet power supply stability over the time interval was good to 1 part in $10^{5}$, leading to probable error of \pm 0.2 Oe for the $(1 / 2,-1 / 2)$ transition; (3) The orientation uncertainty of the $c$ axis of the crystal was $\pm 0.2^{\circ}$ (see section 5.5). This led to an equivalent field uncertainty of \pm 0.1 Oe; (4) Because of the finite time constant of the lock-in amplifier, the recorded signal lags behind the magnetic field. This leads to different values of the resonance line center when the line is traversed with increasing or decreasing magnetic fields. The probable error in the field at the line center from this cause is \pm 0.2 Oe at all fields for the rate of sweep we employed. The error of $g_{\perp}$ is larger, since the errors propagate when eqs (5.4) to (5.6) are used to find the value of $g_{\perp}$.

\subsection{Surface Effects}

It is well known that the intensities of the $(3 / 2,1 / 2),(1 / 2,-1 / 2)$ and $(-1 / 2,-3 / 2)$ transitions when the external magnetic field is parallel to the $c$ axis should be in the ratio of 3:4:3. Experimentally, the measured ratios of the integrated areas of the absorption curves often occur with the $(1 / 2,-1 / 2)$ transition stronger than predicted. The reason offered is that the zero field splitting term, $D$, may have slightly different values for individual crystalline sites, due to crystal imperfections. The $(1 / 2,-1 / 2)$ transition will not be affected by $D$, but the other transitions for those affected sites may have slightly different frequencies, and hence occur at slightly different magnetic fields. This spreading of the $D$ value will then broaden the $(3 / 2,1 / 2)$ and $(-1 /$ $2,-3 / 2)$ lines, and if the spreading is excessive, part of the measured intensity of these lines will be lost. This effect is a hindrance for the use of ruby as a standard since we want to measure the number of spins in terms 
of the line intensities. If the line intensities are not internally consistent with each other, the measurement will be meaningless. It is important to understand the mechanism of the spreading of $D$ in order to minimize the effect or to specify corrections.

The solution turned out to be rather simple. The as-cut surface is essentially amorphous, and the subsurface contains dislocations, plastic deformations, twins, and is heavily strained [21]. Hence, the $\mathrm{Cr}^{3+}$ sites near the surface may have a range of axial crystalline fields and the $D$ value will be spread. The amorphous layer broadens the line so that even the hyperfine line of the $(1 / 2,-1 / 2)$ transition cannot be observed ordinarily. The elaborate procedure of annealing and chemical etching as described was adopted. After the treatment of the samples, the EPR spectrum had improved characteristics. The intensity ratio was 3:4:3 within the experimental error. For our samples the line width (measured between steepest slopes) was typically about 14 Oe for the $(1 / 2,-1 / 2)$ transition, and about 16 Oe for the other transitions. At room temperature the two outer hyperfine lines can be clearly seen for the $(1 / 2,-1 / 2)$ line and can be clearly identified for the other lines as two extended wings.

\subsection{Orientation Uncertainty}

Even if the $c$ axis of the starting boule can be located to within $0.1^{\circ}$, the samples, after cutting and treatment, may have an angular deviation greater than $0.1^{\circ}$. In our case, the maximum deviation is $0.5^{\circ}$. If the sample is mounted in a cavity which is well placed with respect to the magnet, the final angular misalignment should be of the order of $0.5^{\circ}$.

If the crystalline orientation is such that the $c$ axis lies in the horizontal plane, then the magnet (or the sample) can be rotated to line up the $c$ axis with the magnetic field. If the $c$ axis makes a small angle $\delta$ with the horizontal plane, it is always possible to set the magnetic field and the $c$ axis in a vertical plane (see fig. 12). The experimental " $c$-axis data" collected at this $c$-position clearly will have an angular error $\delta$. If the magnet or the sample is rotated away from this $c$-position through an angle $\phi$, the actual angle between the magnetic field and the $c$ axis is $\theta$. From trigonometry

$$
\cos \theta=\cos \delta \cos \phi .
$$

For $\delta=0.5^{\circ}$ and $\phi$ greater than $2^{\circ}, \theta$ will not differ more than $0.1^{\circ}$ from $\phi$. For $\delta=1.0^{\circ}$, the same is true when $\phi \geq 5^{\circ}$. If $\phi=90^{\circ}$, then $\theta=90^{\circ}$ exactly. Thus, a small angular misalignment will not affect the measurements significantly.

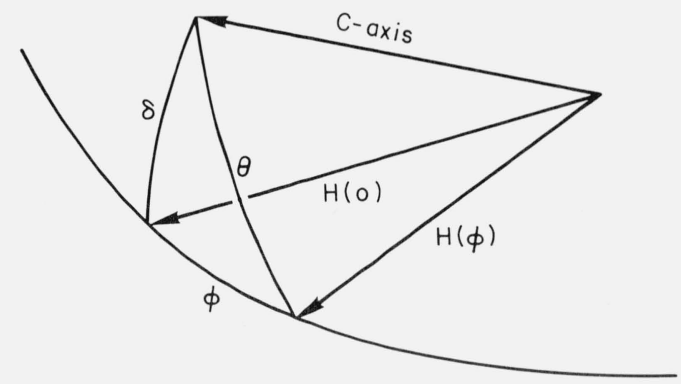

FIGURE 12. The $c$ axis and the rotation of the magnetic field $\mathrm{H}_{0}$.

From the angular dependence diagrams, figure 1 through figure 7 , all curves for the resonance field intersect the $0^{\circ}$ axis with zero slope, i.e., either at a maximum or at a minimum. For X-band frequency, the $(1 / 2,-1 / 2)$ curve is a maximum, hence any angular misalignment will move the transition toward a lower field, and the $g$-value so obtained will be larger than the true value. For K-band frequency, this transition is at a minimum, therefore the $g$-value will be smaller than the true value, if there is angular misalignment. Comparison of these two measurements can be used as a test for the orientation of the $c$ axis. 
The zero slope at $0^{\circ}$ causes uncertainty of the location of the $c$ axis. We have found experimentally at 9.5 $\mathrm{GHz}$ that there is a region of about $0.2^{\circ}$ from the $c$ axis in which the angular variation of the resonance field for the $(1 / 2,-1 / 2)$ transition is not detectable. There is also a region of about $0.5^{\circ}$, where the variation is about 0.1 Oe which is about the limit of field measurement. Although it is still possible to set the magnetic field at the center of this region, the uncertainty of the location of the $c$ axis is $\pm 0.2^{\circ}$. If the magnetic field is rotated off this position by $1^{\circ}$, the center of the $(1 / 2,-1 / 2)$ line will shift about 4 Oe, equivalent to $g_{\|}$being increased by 0.1 percent.

\subsection{Concentration}

The concentration of the $\mathrm{Cr}^{3+}$ in the ruby SRM was measured by static magnetic susceptibility and absolute EPR absorption methods. The ruby samples used in these measurements are described in table 8 . The description of the two methods follows.

(1) Susceptibility Measurement: The detailed theory and the experiment for this type of measurement has been described by Candela and Mundy [22]. A piece of ruby was placed in a magnetic field and the susceptibility was measured. Then the $\mathrm{Cr}^{3+}$ Zeeman levels was saturated by microwave radiation, and the susceptibility was measured again. The difference of the two measurements is the susceptibility due to the $\mathrm{Cr}^{3+}$ system in the sample. From the molar susceptibility of $\mathrm{Cr}^{3+}$, the molar concentration of $\mathrm{Cr}^{3+}$ in the sample could then be obtained. The concentration so measured was taken as the concentration of $\mathrm{Cr}^{3+}$ in the SRM. The value obtained was $(3.694 \pm 0.011) \times 10^{15} \mathrm{Cr}^{3+} / \mathrm{mg}$ of $\mathrm{Al}_{2} \mathrm{O}_{3}$, where the precision quoted is the standard error. This value is believed to be free of systematic error. The susceptibility data from which this result was obtained are shown in table 9.

(2) Absolute EPR Measurement: The $\mathrm{Cr}^{3+}$ concentrations of the SRM samples were determined by static susceptibility. Nevertheless it is important to know that the EPR absorption experiment is sensitive to essentially all the $\mathrm{Cr}^{3+}$ ions in the sample. For this reason we have performed an absolute absorption experiment on several samples. From the results it was verified that within the limit of error, the EPR absorption does indeed arise from all the $\mathrm{Cr}^{3+}$ ions present in the sample.

In the absolute EPR measurement, the energy absorbed by the spin system on resonance is measured and the total number of spins participating in this transition is calculated [23]. The energy absorption rate will depend on the intensity of the radiation field to which the spin system is exposed, and this radiation field is related to the input power, the configuration of the microwave cavity, and the position of the sample in the cavity. These complex relations can be represented by the cavity quality factor $Q$ as defined in eq (2.31), and the energy absorbed on resonance can be deduced from a measurement of the voltage reflection coefficient of the cavity while the applied field $H_{0}$ is adjusted for "off-resonance" and "on-resonance" conditions.

Let the voltage reflection coefficient of the cavity off resonance be denoted by $\Gamma_{0}$ and that on resonance by $\Gamma_{\text {res. }}$. From Feher's treatment [14], modified to account for our use of a circulator instead of a magic tee, we find, for the case where the cavity is critically coupled to the microwave system,

$$
\Delta \Gamma=\Gamma_{\text {res }}-\Gamma_{0}=(1 / 2) \frac{\Delta Q}{Q_{0}}
$$

where $\Delta Q=Q_{0}-Q_{\text {res. }}$. Combining eq (5.10) with (2.37) and (2.38), we have in the neighborhood of a single transition say the $(\alpha, \beta)$ transition,

$$
\Delta \Gamma=N_{0} \frac{2 \pi^{2} Q A \bar{g}^{2} \mu_{B}^{2} \nu}{(2 S+1) k T}\left|\left\langle\alpha\left|S_{l}\right| \beta\right\rangle\right|^{2} \frac{f_{\alpha \beta}\left(H_{0}\right)}{\frac{d \nu_{\alpha \beta}}{d H_{0}}}
$$

where

$$
A=\eta / V_{s}
$$


TABLE 8. Description of samples used in susceptibility, absolute EPR, and integrated intensity measurements

\begin{tabular}{|c|c|c|c|}
\hline Sample Number & Doping Rate (wt. \%) & Shape & Remarks \\
\hline 1 & $0.05 \%$ & round cylinder & $\begin{array}{l}m=290.371 \mathrm{mg}, \text { size about } 3 \times 10 \mathrm{~mm}, \text { from the same boule of } \\
\text { material as SRM's. }\end{array}$ \\
\hline 2 & $.05 \%$ & round cylinder & $m=409.277 \mathrm{mg}$, from the same boule of material as SRM's. \\
\hline 3 & $.05 \%$ & round cylinder & $m=290.35 \mathrm{mg}$, from the same boule of material as SRM's. \\
\hline 4 & $.05 \%$ & cube & size $3 \times 3 \times 3 \mathrm{~mm}$ from the same boule of material as SRM's. \\
\hline 5 & $0.007 \%$ & thin plate & \\
\hline 6 & $0.14 \%$ & thin plate & \\
\hline 7 & $.05 \%$ & bar & size $0.5 \times 0.5 \times 4 \mathrm{~mm}$, SRM control sample. \\
\hline 8 & $.05 \%$ & square & $1.5 \times 1.5 \times 0.5 \mathrm{~mm}, \mathrm{SRM}$ control samples. \\
\hline 9 & $.05 \%$ & thin plate & from the same boule of material as SRM's. \\
\hline
\end{tabular}

TABLE 9. The static susceptibility measurement on sample 1, at $14.5 \mathrm{GHz}$.

Based on $\chi_{m}=\frac{N \mu_{B g^{2}}^{2} S(S+1)}{3 k(T+\theta)}$, where $\theta=0.2 \mathrm{~K}$ for ruby, and $\chi_{m}(T+\theta)=1.8417$ for one g-mol $(52.00 \mathrm{~g})$ of $\mathrm{Cr}$.

\begin{tabular}{|c|c|c|}
\hline$T(\mathrm{k})$ & $\chi_{m}(T+\theta) \times 10^{3}$ & Concentration \\
\hline 4.20 & 0.592 & $3.725 \times 10^{15} \mathrm{Cr}^{3+} / \mathrm{mg}$ of $\mathrm{Al}_{2} \mathrm{O}_{3}$ \\
\hline 3.13 & .587 & 3.683 \\
\hline 2.75 & .592 & 3.725 \\
\hline 2.44 & .583 & 3.668 \\
\hline 2.11 & .590 & 3.701 \\
\hline 2.04 & .584 & Avg. $\quad \frac{3.664}{3.694} \times 10^{15}$ \\
\hline
\end{tabular}

Standard deviation $S_{x}=\sqrt{\frac{\sum_{n}\left(x_{i}-x\right)^{2}}{n-1}}=0.028$

Standard error $S_{\bar{x}}=\frac{S_{x}}{\sqrt{n}}=0.011$

We applied this analysis to the $(1 / 2,-1 / 2)$ transition of $\mathrm{Cr}^{3+}$ in ruby at a microwave frequency of $9.5 \mathrm{GHz}$ and orientation $\theta=0^{\circ}$. The weighting factor for this case is

$$
\frac{d \nu_{1 / 2,-1 / 2}}{d H_{0}}=\frac{g_{\|} \mu_{B}}{h}
$$

and the matrix element has the value unity. Hence, the number of $\mathrm{Cr}^{3+}$ can be obtained from eq. (5.11) in the form

$$
N_{0}=\frac{2 k T \Delta \Gamma}{\pi^{2} \bar{g} \mu_{B} h \nu f_{1 / 2,-1 / 2}\left(H_{0}\right) Q_{0} A},
$$

where for this application, the difference between $g_{\|}$and $g_{\perp}$ is neglected. In (5.14), besides physical constants, $T$ and $\nu$ can be measured during experiment. We have $A, f_{1 / 2,-1 / 2}\left(H_{0}\right), Q_{0}$ and $\Delta \Gamma$ to be determined. 
The filling factor of a small sample placed at the center of a cylindrical cavity of radius $a$ and height $d$ is [15]

$$
\eta=\frac{12.33}{1+(0.82 a / d)^{2}} \frac{V s}{V_{c}}
$$

hence,

$$
A=\frac{12.33}{1+(0.82 a / d)^{2}} \frac{1}{V_{c}}
$$

In our case, the X-band cavity is $4.148 \mathrm{~cm}$ in diameter, and $4.161 \mathrm{~cm}$ high, hence,

$$
A=0.1879 \text {. }
$$

We apply eq (5.14) at the absorption peak, hence $f_{1 / 2,-1 / 2}\left(H_{0}\right)$ becomes $F_{0}\left(H_{0}\right)$ which is the peak height of the line shape function when the area under the line shape function is normalized to unity. The peak height therefore, should be independent of the particular cavity and individual samples as long as they have the same $\mathrm{Cr}^{3+}$ concentration. The $(1 / 2,-1 / 2)$ transition was observed several times at room temperature with different samples and different cavities. Areas were found by graphical integration and also by computer analysis. The final result is

$$
f_{0}\left(H_{0}\right)=0.0505
$$

Before we could attempt to measure $Q_{0}$ and $\Delta \Gamma$ the microwave system had to be calibrated. The microwave frequency was set at the center frequency of the sample cavity. Referring to the block diagram of figure 8, let the location of the cavity be called position A and the location of the power meter \#2 be called position B. First, the cavity and the iris were removed and the thermistor of power meter \# 2 was connected to the waveguide at position $\mathrm{A}$. We could then measure the microwave power that was normally incident on the cavity. This power was measured against various settings of the attenuator (while power meter \# 1 was kept at a definite level, in our case $0.1 \mathrm{~mW}$ ). Then the waveguide at position A was shorted with a piece of copper plate, so the power incident on it would be reflected totally. The power meter \#2 was connected at its normal position B. Since there were insertion losses in the waveguide, the circulator, and the waveguide switch, the reading of power meter \#2 at B was not the same as it was at A. However, the reading at B gave the actual real reflected power at $\mathrm{A}$.

The cavity $Q$ was measured in the following manner. The cavity was first adjusted to critical coupling, and power meter \#2 read the reflected power which was usually very small, but not exactly equal to zero. Then the frequency was varied by fine tuning of the frequency stabilizer, and the reflection was measured. The response curve was plotted and the $3 \mathrm{~dB}$ (half power) width, $\Delta \nu$, was measured. Then $Q$ was calculated by

$$
Q_{L}=\nu_{0} / \Delta \nu
$$

The $Q$ measured in this way is the loaded $Q$, and is related to $Q_{0}$ by

$$
\frac{1}{Q_{L}}=\frac{1}{Q_{0}}+\frac{1}{Q_{\mathrm{ext}}} .
$$

At critical coupling $Q_{0}=Q_{\text {ext }}$. Hence, under this condition

$$
Q_{0}=2 Q_{L}
$$

For an X-band cylindrical cavity of $\mathrm{TE}_{011}$ mode, at room temperature, the $Q_{L}$ is typically about 12,000 , the half width is typically about $0.8 \mathrm{MHz}$. 
The $\Delta \Gamma$ measurement was performed as follows. The magnetic field modulation was turned off. The field sweep was driven slowly from the off resonance condition toward resonance. The power input and power reflected were noted. As the magnetic field moved into the resonance line, the reflected power started to change. If the resonance line were traversed and the power meter readings were plotted against the corresponding magnetic field, the plot would display the line shape function $f\left(H_{0}\right)$. The maximum power reflected is the on-resonance reflection at the peak of the EPR absorption. The power reflection ratios were evaluated; the square root of the power reflection ratio is the voltage reflection ratio. The difference of voltage reflection ratios is the $\Delta \Gamma$ to be measured. $\Delta \Gamma$ was measured repeatedly with different input power and different sweep speed. Several samples were measured. The samples were cut from the same material as the SRM's, but were of larger size to produce measurable reflection at resonance. Typically, at an input power level of $1 \mathrm{mw}$, the reflection off resonance is essentially zero, that on resonance is about $1 \mu \mathrm{W}$ for a $10^{18}$ spin sample. The value of concentration obtained from these measurements are listed in table 10 . The variability between the three averages is characterized by a coefficient of variation of 5 percent. To within this uncertainty, the values are in good agreement with that obtained by susceptibility measurements. This method gives the total concentration of $\mathrm{Cr}^{3+}$ including the isotopic fraction possessing nuclear moments. This is true because the normalized line shape function used includes the contributions of the unresolved hyperfine components.

TABLE 10. Summary of the absolute EPR absorption measurements Both the average concentration and the standard deviation are in units of $10^{15} \mathrm{Cr}^{3+}$ ions $/ \mathrm{mg} \mathrm{of} \mathrm{Al}_{2} \mathrm{O}_{3}$.

\begin{tabular}{c|c|c|c}
\hline \hline Sample Number & No. of Observations & Average Concentration & Standard Deviation \\
\hline 1 & 11 & 3.79 & 0.49 \\
2 & 16 & 3.55 & .41 \\
\hline
\end{tabular}

Mean of averages $=3.75 \times 10^{15} \mathrm{Cr}^{3+} / \mathrm{mg}$ of $\mathrm{Al}_{2} \mathrm{O}_{3}$.

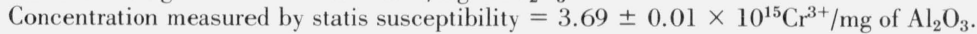

\subsection{Integrated Intensities}

The integrated intensity has been defined (see sec. 2.6) as the area under the resonance absorption curve. To obtain the integrated intensity experimentally, it is necessary to establish a base line for the absorption curve. The integrated intensity of the different transitions should be proportional to the corresponding $U$ factors listed in tables $1-7$.

The modulation technique generally used for the detection of the EPR signal yields the first derivative of the absorption curve. The first derivative can be integrated once to restore the absorption curve; the area can then be obtained by a second integration. Ideally, the first derivative should be zero when the magnetic field is set beyond the resonance line. For a narrow line, the zero background level usually can be determined easily. Even if there is uncertainty about where the zero level is, the error introduced is usually small. For a broad line, there is always doubt as to where the zero level really begins. The error thus introduced will be sizable, since the contribution from the wings after integration is appreciable. After the first integration, if the restored absorption curve is plotted against the magnetic field, the base line is usually not horizontal. This is the so-called base line drift. During the second integration, correction is necessary to compensate for this drift.

We measured the integrated intensity at $9.5 \mathrm{GHz}$ at $y^{\prime}$-polarization. At $\theta=0^{\circ}$ and $\theta=90^{\circ}$, the EPR lines are reasonably narrow and easily measured. The integrated intensities were normalized with respect to the measured area of the $(1 / 2,-1 / 2)$ transition. The normalized intensities are shown in table 11 . The deviation from the theoretical values, which are listed in the table, varied up to 6 percent. For the intermediate orientations, at steps of ten degrees, all the transitions with $U_{y^{\prime}}$ greater than 0.250 were measured. Except for three "bad" lines, the agreement between the measured integrated intensity and the calculated $U$-factors was \pm 10 percent. The three bad lines have large $U$-factors, but they are very broad. Hence, the experimentally measured integrated intensities have much larger errors. Sometimes the discrepancy between the measured 
integrated intensity and the $U$-factor was as great as \pm 50 percent. These lines are the $(1,2)$ transition at $30^{\circ}$, and again at $40^{\circ}$, and the $(2,3)$ transition at $70^{\circ}$ at 3143.7 Oe.

TABLE 11. Tabulation of measured intensities at $0^{\circ}$ and $90^{\circ}$ Data are normalized to the value of the $(1 / 2,-1 / 2)$ transition at $0^{\circ}$.

\begin{tabular}{|c|c|c|c|c|}
\hline \multirow{2}{*}{ Angle $\theta$} & \multicolumn{2}{|c|}{$0^{\circ}$} & \multicolumn{2}{|c|}{$90^{\circ}$} \\
\hline & $(3 / 2,1 / 2)$ & $(-1 / 2,-3 / 2)$ & $(1,2)$ & $(3,4)$ \\
\hline $\begin{array}{l}\text { Theoretical } \\
\text { Intensity, } U_{y^{\prime}}\end{array}$ & 0.750 & 0.750 & 0.802 & 0.470 \\
\hline Sample \# 1 & .785 & & & \\
\hline Sample \#4 & .735 & & & \\
\hline Sample \#4 $4^{\text {a }}$ & $\begin{array}{l}.758 \\
.743\end{array}$ & $\begin{array}{l}.750 \\
.713\end{array}$ & $\begin{array}{l}.848 \\
.840\end{array}$ & $\begin{array}{l}.498 \\
.495\end{array}$ \\
\hline Sample \#5 & $\begin{array}{l}.779 \\
.761\end{array}$ & & & \\
\hline Sample \#6 & .777 & & & \\
\hline
\end{tabular}

a This set of measurements were performed by us on an EPR spectrometer at the National Institutes of Health. All other measurements in this table were performed at NBS.

TABLE 12. Tabulation of measured intensities for values of $\theta$ from $0^{\circ}$ to $90^{\circ}$.

Intensities are normalized to the measured area of the $(1 / 2,-1 / 2)$ transition. The theoretical values $U_{y^{\prime}}$ are listed for the various transitions. When a particular transition is observed at two field values, the respnance is identified as high (hi) or low (lo) field. The asterisk identifies broad resonance lines for which a precise intensity measurement is difficult.

\begin{tabular}{|c|c|c|c|c|c|c|c|}
\hline$\checkmark \theta$ & Transitions & $U_{y^{\prime}}$ & Sample \#7 & Sample \#8 & Sample \#4 & Sample \#9 & Sample \#9† \\
\hline $0^{\circ}$ & $\begin{array}{l}(1.3) \mathrm{Lo} \\
(1.3) \mathrm{Hi} \\
(1.2)\end{array}$ & $\begin{array}{l}0.750 \\
1.000 \\
0.750\end{array}$ & $\begin{array}{r}0.698 \\
.708\end{array}$ & $\begin{array}{r}0.710 \\
.680\end{array}$ & $\begin{array}{r}0.720 \\
.728\end{array}$ & 0.725 & \\
\hline $10^{\circ}$ & $\begin{array}{l}\text { (1.3) Lo } \\
(1.3) \mathrm{Hi} \\
(2.3) \\
(1.2)\end{array}$ & $\begin{array}{l}.768 \\
.600 \\
.358 \\
813\end{array}$ & $\begin{array}{l}.695 \\
.680 \\
.320 \\
.753\end{array}$ & $\begin{array}{l}.708 \\
.665 \\
.313 \\
.743\end{array}$ & $\begin{array}{l}.988 \\
.638 \\
.368 \\
.845\end{array}$ & $\begin{array}{l}.558 \\
.325 \\
.720\end{array}$ & \\
\hline $20^{\circ}$ & $\begin{array}{l}\text { (1.3) Lo } \\
(1.3) \mathrm{Hi} \\
(2.3) \\
(1.2)\end{array}$ & $\begin{array}{r}.861 \\
.299 \\
.495 \\
1.076\end{array}$ & $\begin{array}{r}.835 \\
.345 \\
.473 \\
1.045\end{array}$ & $\begin{array}{r}.803 \\
.315 \\
.485 \\
1.033\end{array}$ & $\begin{array}{r}.903 \\
.273 \\
.485 \\
1.250\end{array}$ & $\begin{array}{r}.325 \\
.493 \\
1.210\end{array}$ & \\
\hline $30^{\circ}$ & $\begin{array}{l}*(1.2) \\
\quad(2.3) \mathrm{Hi}\end{array}$ & $\begin{array}{l}2.032 \\
0.568\end{array}$ & $\begin{array}{l}1.650 \\
0.545\end{array}$ & $\begin{array}{l}1.053 \\
0.543\end{array}$ & $\begin{array}{r}1.833 \\
.588\end{array}$ & $\begin{array}{l}2.295 \\
0.575\end{array}$ & \\
\hline $40^{\circ}$ & $\begin{array}{l}*(1.2) \\
\quad(2.3) \mathrm{Hi}\end{array}$ & $\begin{array}{l}1.828 \\
0.634\end{array}$ & $\begin{array}{l}2.258 \\
0.610\end{array}$ & $\begin{array}{l}1.925 \\
0.598\end{array}$ & $\begin{array}{r}1.535 \\
.653\end{array}$ & $\begin{array}{l}1.720 \\
0.595\end{array}$ & \\
\hline $50^{\circ}$ & $\begin{array}{l}(1.2) \\
(2.3) \mathrm{Hi}\end{array}$ & $\begin{array}{l}1.160 \\
0.717\end{array}$ & $\begin{array}{l}1.088 \\
0.668\end{array}$ & $\begin{array}{l}1.100 \\
0.665\end{array}$ & $\begin{array}{r}1.295 \\
.758\end{array}$ & $\begin{array}{l}1.270 \\
0.735\end{array}$ & $\begin{array}{r}0.980 \\
.645\end{array}$ \\
\hline $60^{\circ}$ & $\begin{array}{l}(2.3) \mathrm{Lo} \\
(1.2) \\
(3.4) \\
(2.3) \mathrm{Hi}\end{array}$ & $\begin{array}{l}.254 \\
.953 \\
.276 \\
.847\end{array}$ & $\begin{array}{l}.258 \\
.923 \\
.265 \\
.825\end{array}$ & $\begin{array}{l}.258 \\
.908 \\
.255 \\
.760\end{array}$ & $\begin{array}{r}.273 \\
1.023 \\
.265 \\
.855\end{array}$ & $\begin{array}{l}.988 \\
.263 \\
.765\end{array}$ & $\begin{array}{l}.768 \\
.208 \\
.708\end{array}$ \\
\hline $70^{\circ}$ & $\begin{aligned} & (2.3) \mathrm{Lo} \\
& (1.2) \\
* & (2.3) \mathrm{Hi} \\
& (3.4)\end{aligned}$ & $\begin{array}{r}.386 \\
.860 \\
1.087 \\
0.465\end{array}$ & $\begin{array}{l}.363 \\
.828 \\
.960 \\
.383\end{array}$ & $\begin{array}{r}.363 \\
.815 \\
1.540 \\
0.338\end{array}$ & $\begin{array}{r}.408 \\
1.258 \\
1.078 \\
.448\end{array}$ & & $\begin{array}{l}.740 \\
.863 \\
.320\end{array}$ \\
\hline $80^{\circ}$ & $\begin{array}{l}(2.3) \mathrm{Lo} \\
(1.2) \\
(2.3) \mathrm{Hi} \\
(3.4)\end{array}$ & $\begin{array}{r}.784 \\
.815 \\
1.769 \\
0.478\end{array}$ & $\begin{array}{r}.820 \\
.773 \\
1.330 \\
.390\end{array}$ & $\begin{array}{r}.830 \\
.775 \\
1.718 \\
0.438\end{array}$ & $\begin{array}{r}.738 \\
.870 \\
1.765 \\
.500\end{array}$ & & $\begin{array}{r}.825 \\
1.705 \\
0.393\end{array}$ \\
\hline $90^{\circ}$ & $\begin{array}{l}(1.2) \\
(3.4) \\
\end{array}$ & $\begin{array}{l}.802 \\
.470 \\
\end{array}$ & $\begin{array}{l}.795 \\
.460 \\
\end{array}$ & $\begin{array}{l}.750 \\
.440 \\
\end{array}$ & $\begin{array}{l}.870 \\
.528\end{array}$ & & .453 \\
\hline
\end{tabular}

Note: Measurements on sample \#7, 8, and 4 were performed by us on an EPR spectrometer at the National Institutes of Health. Measurements on sample \#9 were performed at NBS.

$\dagger$ These measurements are normalized to the measured areas of (1.2) transition at $90^{\circ}$. 
The results of five sets of measurements on four samples for angles varying from $0^{\circ}$ to $90^{\circ}$ in steps of $10^{\circ}$ are listed in table 12. As before, the integrated areas are normalized relative to the $(1 / 2,-1 / 2)$ transition at $0^{\circ}$, except the second measurement for sample \#9, which was normalized relative to $(1,2)$ transition at $90^{\circ}$. The theoretical values of $U_{y}$ are listed for comparison. If we omit the broad lines, of 106 relative intensity measurements, 82 differ from the theoretical values by less than 10 percent, 18 differ by an amount between 10 percent and 20 percent, and 6 differ by more than 20 percent.

The authors wish to take this opportunity to express their gratitude to many collegues at NBS who offered considerable help during the course of this research. They wish to extend special thanks to Dr. George Candela for performing the static susceptibility measurements, to Dr. Norman Wolcott and Dr. Robert J. Arms for advice on computational procedures, to Dr. Bernard J. Hockey and Dr. Nancy Tighe for advice on surface damage problems and treatment, to Dr. Richard A. Forman for numerous suggestions and literature citations, to Ms. Mary Heasley for data taking and reduction, and finally to Dr. Herbert S. Bennett for continuous encouragement. Dr. Edmund Copeland of the National Institutes of Heaith generously let us test samples with his EPR equipment. Lastly, the authors are grateful for helpful advice on statistical analysis from Dr. John Mandel and Dr. Robert C. Paule.

\section{Appendix}

A short explanation of the units used in this publication follows. The energy of an atomic magnetic moment in an applied magnetic field is determined by the magnetic field strength $H$. The most commonly used unit of $H$ in the practice of magnetic resonance is the c.g.s. electromagnetic unit, the oersted, abbreviated Oe. Accordingly we use the oersted in this report. The magnetic induction $B$ is measured in the c.g.s. electromagnetic system in gauss, abbreviated G. For this system of units, in vacuum $B$ has a numerical value equal to the value of the field strength $H$, and the two are sometimes referred to interchangeably. The SI (Systémè International) unit of $H$ is the ampere/meter $(\mathrm{A} / \mathrm{m})$ and that of $B$ is the tesla $(\mathrm{T})$. The quantities of $H$ and $B$ may be converted from c.g.s. electromagnetic units to SI units by the formulas:

$$
\begin{gathered}
H(A / m)=H(O e) \times 10^{3} / 4 \pi \\
B(T)=B(G) \times 10^{-4} .
\end{gathered}
$$

\section{References}

[1] Singer, L. S., J. Appl. Phys. 30,1463 (1959).

[2] Manenkov, A. A., and Prokhorov, A. M., Zh. Eksp. Teor. Fiz. 28, 762 (1955); [translation: Soviet Phys. JETP 1, 611 (1955)]

[3] Geusic, J. E., Phys. Rev. 102, 1252 (1956).

[4] Zaripov, M. M., and Shamonin, Iu. Ia., Zh. Eksp. Teor. Fiz. 30, 2911 (1956), [translation: Soviet Phys. JETP 3, 171 (1956)]

[5] Kikuchi, C., Lambe, J., Makhov, G., and Terhune, R. W., J. Appl. Phys. 30, 1061 (1959).

[6] Statz, H., and Koster, G. F., Phys. Rev. 115, 1568 (1959).

[7] Chang, W. S., private communication.

[8] Schulz-du Bois, E. O., Bell System Tech. J. 38, 271 (1959).

[9] Schiff, L. I., Quantum Mechanics (McGraw-Hill, 1949). See Chap. X.

[10] See ref. 9, Chap. VI.

[11] Condon, E. U., and Shortley, G. H., The Theory of Atomic Spectra (Cambridge, 1953). See Chap. III.

[12] Feynman, R. P., Phys. Rev. 56, 340 (1939).

[13] Hellmann, H., Quantenchemie, Deuticke \& Co., Leipzig (1937). See p. 285.

[14] Feher, G., Bell System Technical Journal, 36, 449 (1957).

[15] Poole, C. P., Jr., Electron Spin Resonance (Interscience, 1967). See p. 291.

[16] Bloembergen, N., Purcell, E. M., and Pound, R. V., Phys. Rev. 73, 679 (1948).

[17] Blunt, R. F., private communication.

[18] Charvat, F. R., Smith, J. C., and Nestor, O. H., Proc. Int. Conf. on Crystal Growth, Boston, 20-24 June, 1966. Suppl. to J. Phys. Chem. Solids, p. 45 (1967). 
[19] Ensign, T. C., and Chang, T., Rev. Sci. Instrum. 40, 268 (1969).

[20] Gordon, J. P., Rev. Sci. Instrum. 32, 658 (1961).

[21] Hockey, B. J., The Science of Ceramic Machining and Surface Finishing, Proceedings of a Symposium, Nat. Bur. Stand. (U.S.), Spec. Publ. 348, 431 pages (May 1972).

[22] Candela, G. A., and Mundy, R. E., Rev. Sci. Instrum. 36, 338 (1968).

[23] Yariv, A., and Gordon, J. P., Rev. Sci. Instrum. 32, 462 (1961). 